\title{
The Syenite-Carbonatite Complex of
} lhouhaouene (Western Hoggar, Algeria): Interplay Between Alkaline Magma Differentiation and Hybridization of Cumulus Crystal Mushes

\section{OPEN ACCESS}

Edited by:

Michel Grégoire,

Center National de la Recherche

Scientifique (CNRS), France

Reviewed by:

Theo Ntaflos,

University of Vienna, Austria

Michael Anenburg,

Australian National University,

Australia

*Correspondence:

A. Djeddi

djeddiasmageo/@gmail.com

Specialty section:

This article was submitted to

Geochemistry,

a section of the journal

Frontiers in Earth Science

Received: 11 September 2020

Accepted: 11 December 2020

Published: 04 February 2021

Citation:

Djeddi A, Parat F, Bodinier J-L, Ouzegane K and Dautria J-M (2021) The Syenite-Carbonatite Complex of Ihouhaouene Western Hoggar, Algeria): Interplay Between Alkaline

Magma Differentiation and Hybridization of Cumulus

Crystal Mushes.

Front. Earth Sci. 8:605116. doi: 10.3389/feart.2020.605116

\section{A. Djeddi ${ }^{1,2 *}$, F. Parat ${ }^{1}$, J.-L. Bodinier ${ }^{1,3}$, K. Ouzegane ${ }^{2}$ and J.-M. Dautria ${ }^{1}$}

${ }^{1}$ Géosciences Montpellier, UMR 5243, CC60, Université de Montpellier, 34095 Montpellier Cedex 5, France, ${ }^{2}$ FSTGAT-USTHB, BP32, El Alia 16111 Bab Ezzouar, Algeria, ${ }^{3}$ Mohammed VI Polytechnic University, Geology and Sustainable Mining, Ben Guerir, Morocco

The 2 Ga-old lhouhaouene alkaline complex (Western Hoggar, Algeria) is among the oldest known carbonatite occurrences on Earth. The carbonatites are calciocarbonatites hosted by syenites, the predominant rock type in the complex. Both rock types are characterized by medium-grained to pegmatitic textures and contain clinopyroxene, apatite, and wollastonite, associated with K-feldspar in syenites and a groundmass of calcite in carbonatites. The rock suite shows a continuous range of compositions from 57-65 wt.\% $\mathrm{SiO}_{2}$ and $0.1-0.4$ wt.\% $\mathrm{CO}_{2}$ in red syenites to $52-58 \mathrm{wt} . \% \mathrm{SiO}_{2}$ and 0.1-6.5 wt.\% $\mathrm{CO}_{2}$ in white syenites, $20-35$ wt. $\% \mathrm{SiO}_{2}$ and $11-24$ wt. $\% \mathrm{CO}_{2}$ in Si-rich carbonatites (>10\% silicate minerals), and $<20$ wt. $\% \mathrm{SiO}_{2}$ and $24-36 \mathrm{wt.} \% \mathrm{CO}_{2}$ in Si-poor carbonatites ( $<5 \%$ silicate minerals). Calculation of mineral equilibrium melts reveals that apatite and clinopyroxene are in disequilibrium with each other and were most likely crystallized from different parental magmas before being assembled in the studied rocks. They are subtle in the red syenites, whereas the white syenites and the Si-rich carbonatites bear evidence for parental magmas of highly contrasted compositions. Apatite was equilibrated with LREE-enriched (Ce/Lu $=1,690-6,182)$ carbonate melts, also characterized by elevated $\mathrm{Nb} / \mathrm{Ta}$ ratio $(>50)$, whereas clinopyroxene was precipitated from silicate liquids characterized by lower LREE/HREE (Ce/Lu = 49-234) and variable Nb/ Ta ratios $(\mathrm{Nb} / \mathrm{Ta}=2-30)$. The Si-poor carbonatites resemble the Si-rich carbonatites and the white syenites with elevated REE contents in apatite equilibrium melts compared to clinopyroxene. However, apatite equilibrium melt in Si-poor carbonatite shows a majority of subchondritic values $(\mathrm{Nb} / \mathrm{Ta}<10)$ and clinopyroxene has chondritic-to-superchondritic values $(\mathrm{Nb} / \mathrm{Ta}=15-50)$. Although paradoxical at first sight, this $\mathrm{Nb}$-Ta signature may simply reflect the segregation of the carbonatite from highly evolved silicate melts characterized by extremely low $\mathrm{Nb} / \mathrm{Ta}$ values. Altogether, our results suggest an evolutionary scheme whereby slow cooling of a silico-carbonated mantle melt resulted in the segregation of both cumulus minerals and immiscible silicate and carbonate melt 
fractions, resulting in the overall differentiation of the complex. This process was however counterbalanced by intermingling of partially crystallized melt fractions, which resulted in the formation of hybrid alkaline cumulates composed of disequilibrium cumulus phases and variable proportions of carbonate or K-feldspar.

Keywords: carbonatite, syenite, hybrid mineral aggregates, alkaline magma, Hoggar

\section{INTRODUCTION}

Petrological and geochemical studies supported by experimental works have suggested the origin of carbonatites via two main processes: 1) Partial melting of carbonate-rich mantle producing primary carbonate melts (Gittins and Harmer, 1997; Gudfinnsson and Presnall, 2005; Stoppa et al., 2005; Dasgupta and Hirschmann, 2006). Carbonatites generated by this mechanism are either (1a) magnesio-carbonatites, considered to be efficiently segregated and extracted from the mantle (Gittins and Harmer, 1997; Dalton and Presnall, 1998; Wyllie and Lee, 1998; Hammouda and Keshav, 2015) or (1b) calciocarbonatites, formed by reaction of volatile-rich carbonate melts with depleted lherzolites or harzburgites at low pressure (Dalton and Wood, 1993; Gittins and Harmer, 1997), or by partial melting of $\mathrm{CO}_{2}$-rich eclogites (Hammouda, 2003; Hammouda and Keshav, 2015). 2) Igneous differentiation of carbonate-rich silicate melt leading to unmixing of silicate melt fractions (Freestone and Hamilton, 1980; Baker and Wyllie, 1990; Dawson, 1998; Dasgupta et al., 2006; Brooker and Kjarsgaard, 2011; Baudouin et al., 2016; Weidendorfer et al., 2016). Extreme fractional crystallization of alkaline magmas may also culminate in the segregation of carbohydrothermal carbonatites crystallized at subsolidus temperatures from a mixed $\mathrm{CO}_{2}-\mathrm{H}_{2} \mathrm{O}$ fluid (Woolley and Kjarsgaard, 2008; Mitchell, 2009). In addition, carbonate melts (igneous or unmixed carbonate melt) may be partly counterbalanced by wall-rock assimilation or "antiskarn metasomatism" at high temperature and high pressure, resulting in substantial silica enrichment of carbonatites (Chakhmouradian et al., 2008; Anenburg and Mavrogenes, 2018; Giebel et al., 2019; Anenburg et al., 2020).

However, whatever process is envisioned for the origin of carbonatites, the common association of carbonatites with alkaline silicate rocks must be taken into account as it is key stone to the understanding of their petrogenesis. Most often, carbonatites are associated with silica-undersaturated, highly alkaline silicate rocks such as nephelinites, ijolites, phonolites, and nepheline syenites (e.g., Bell et al., 1998; Woolley, 2003; Woolley and Kjarsgaard, 2008). The association of carbonatites with silica-saturated rocks is less common; it was estimated to represent $8 \%$ of carbonatites worldwide by Woolley and Kjarsgaard (2008).

The Ihouhaouene alkaline complex is one of those. Similar to Mountain Pass, California (Olson et al., 1954), Oka, Canada (Eby, 1975), and Eden Lake, Canada (Chakhmouradian et al., 2008), the complex shows the association of calciocarbonatites with silicasaturated syenites. The two rock types are closely associated in the field, being intermingled at various scales and showing similar pegmatitic/brecciated textures. Carbonatites and syenites also have similar $\mathrm{Nd}, \mathrm{Sr}$, and $\mathrm{C}$ isotopic signatures consistent with a mantle origin and ruling out significant crustal contamination (Bernard-Griffiths et al., 1988; Ouzegane et al., 1988; Fourcade et al., 1996). The rocks are also virtually free from subsolidus reactions and secondary alteration, making the Ihouhaouene complex a valuable case study to address petrogenetic relationships between carbonatites and silica-saturated syenites.

We report major and trace element data for whole rocks and minerals in all rock facies. However, our interpretations are principally based on the systematics of apatite and clinopyroxene equilibrium melts since the whole rocks contain a significant cumulate component and are not considered to represent melt compositions. Our results reveal that carbonatites and syenites from the Ihouhaouene complex are even more intimately related than suggested by geologic, isotopic, and petrographic evidence. The two rock types share a complex history of igneous differentiation partly counterbalanced by mixing of cumulus minerals crystallized from melt fractions of contrasting compositions, including previously segregated carbonate and silicate liquids.

\section{GEOLOGICAL SETTING AND FIELD RELATIONSHIPS}

The Ihouhaouene area is located in the northern part of In Ouzzal terrane (Western Hoggar, south of Algeria; $23^{\circ} 36^{\prime} 14^{\prime \prime} \mathrm{N}$, $\left.3^{\circ} 10^{\prime} 32^{\prime \prime} \mathrm{E}\right)$ and contains carbonatite bodies associated with syenites that are among the oldest in the world $(\sim 2 \mathrm{Ga}$, Bernard-Griffiths et al., 1988). The In Ouzzal terrane is an Archaean crust remobilized during the paleoproterozoic ultrahigh-temperature metamorphism $\left(2 \mathrm{Ga}, \mathrm{T}=800-1,050^{\circ} \mathrm{C}\right.$ at 10-11 kbar, Ouzegane et al., 2003). Two Archaean units occur: a lower crustal unit mainly composed of orthogneiss with igneous activity at $3.2 \mathrm{Ga}$ (Peucat et al., 1996) and a supracrustal unit deposited at 2.6 to $2.7 \mathrm{Ga}$ (Peucat et al., 1996) with quartzites, banded iron formations, marbles, and $\mathrm{Al}-\mathrm{Mg}$ and $\mathrm{Al}-\mathrm{Fe}$ granulites associated with mafic and ultramafic rocks (Guiraud et al., 1996; Peucat et al., 1996; Ouzegane et al., 2003; Adjerid et al., 2013). The In Ouzzal terrane is a stable craton since the Eburnean tectono-metamorphic event (2 Ga, Bernard-Griffiths et al., 1988). The Ihouhaouene carbonatites and syenites are not metamorphosed, and the magmatic characteristics of igneous rocks have been exceptionally well preserved (Bernard-Griffiths et al., 1988; Ouzegane et al., 1988).

The carbonatites and syenites occur as linear outcrops along shear zones in three sectors (Ouzegane et al., 1988; Lapin and Ploshko, 1988; Fourcade et al., 1996; Figure 1): 1) Oued 


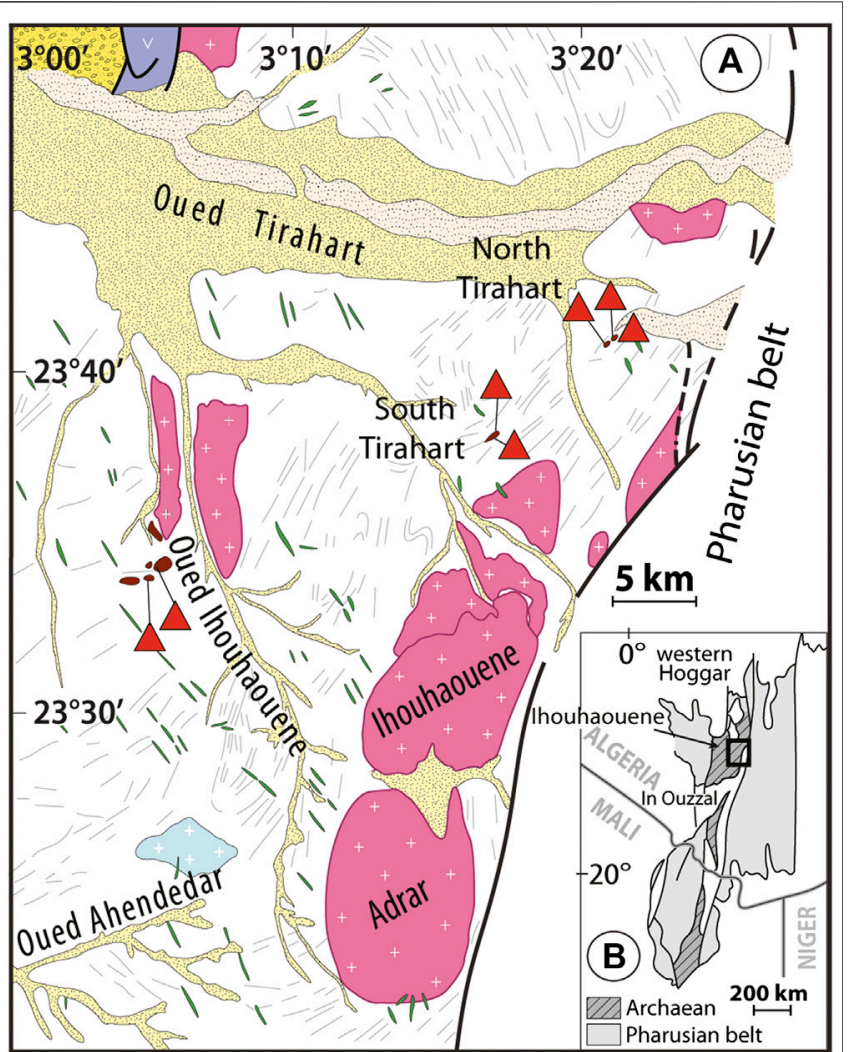

\begin{tabular}{l}
\hline In'Ouzzal granulite \\
Fyenite \\
FIGURE 1 I (A) Geological map of the Ihouhaouene area in NW part of \\
the In Ouzzal terrane and localization of carbonatites and associated syenites \\
(modified after Ouzegane et al., 1988). (B) Position of the Ihouhaouene area in \\
Western Hoggar, south of Algeria (Black et al., 1994).
\end{tabular}

Ihouhaouene characterized by several small hills-oriented EW and NW-SE; 2) the South Tirahart wadi complex with outcrops of approximately $4 \mathrm{~km}$ length and $1 \mathrm{~km}$ width, and 3) Tirahart wadi north, a hill spreading over $2.5 \mathrm{~km}$ length and $250 \mathrm{~m}$ width. Carbonatites outcrop as veins or massive formations in syenites. The contact between carbonatite and syenite is sharp (with no reactional selvage), and only local syenites have mylonitized edges $(<20 \mathrm{~cm})$.

According to their texture, carbonatites present as massive structures up to $50 \mathrm{~m}$ thickness and max. $500 \mathrm{~m}$ length with clinopyroxene and apatite medium grains in calcite matrix and can be brecciated including some syenite fragments with sizes ranging from 1 to $70 \mathrm{~cm}$ partially surrounded by clinopyroxene reaction aureoles (Figures $2 \mathrm{~A}, \mathbf{B}$ ) or as pockets or small veins ( $<2 \mathrm{~m}$ width and $<10 \mathrm{~m}$ length) coarse grained to pegmatitic with euhedral apatite (3-50 mm) (Figures 2C,D) and calcite. Clinopyroxene and alkali feldspar euhedral megacrysts are present in these pegmatitic carbonatites (Figure 2D).

The syenites form large outcrops in all three sectors. They are red (Figure 2E) or white (Figure 2F) according to the presence of red alkali feldspar, or white alkali feldspar and wollastonite, respectively. Both syenites present structures with magmatic segregation of clinopyroxene and feldspar minerals with the same orientation as the surrounding granulites. Red syenites are more abundant in Oued Ihouhaouene and Tirahart wadi North complexes. In Tirahart wadi North complex, white syenite fragments composed of wollastonite, garnet, and large crystals of pyroxene cemented by feldspars form feldspathic breccia. The contact between red and white syenites is brecciated. Only in Oued Ihouhaouene, sharp contacts are observed, with large white syenite bodies crosscutting red syenites (previously mapped as granites by Fourcade et al., 1996). The contact between syenites and granulitic basement is not obvious because of sand cover in this area, but the outcropping granulites (far from $\sim 20 \mathrm{~m}$ ) in Oued Ihouhaouene and North wadi Tirahart present no evidence of fenitization. The granulitic crust preserved refractory chemical compositions (Ouzegane et al., 2003), not consistent with metasomatism and fenitization induced by a second local fluid (e.g., in situ alkali-metasomatism of country rock around intrusions of carbonatites and/or alkaline rocks, Kresten, 1988; Drüppel et al., 2004; Elliott et al., 2018). Syenite outcrops have homogenous mineralogy from the contact with carbonatite to the sand cover area separating the complex from granulite host rock. The sand cover area between syenites and granulite outcrops is not larger than $20 \mathrm{~m}$ in between outcrops and no fenitization aureole has been observed.

\section{PETROGRAPHY}

\section{Carbonatites}

Carbonatites from Ihouhaouene outcrop as pegmatitic veins or pockets with large crystals or brecciated with medium- to coarsegrained textures $(3-8 \mathrm{~mm})$. All carbonatites have euhedral and unzoned apatite and clinopyroxene phenocrysts set in a matrix of primary calcite (50-80 vol.\%, Ouzegane et al., 1988), and they can be defined as calciocarbonatite (Streckeisen, 1974). Whether they outcrop as pegmatitic veins and brecciated, carbonatites have variable mineral abundance (Supplementary Figures S1, S2) with high abundance of silicate minerals (10-20 vol.\%) or low abundance of silicate minerals $(<5 \mathrm{vol} . \%)$, with specific mineral compositions (see below). These carbonatite groups are later called Si-rich and Sipoor carbonatites (major mineralogy in Supplementary Table S1).

Si-poor carbonatites (Figure 3A) have clinopyroxene $(<2 \mathrm{~mm}$, 1 to 5 vol.\%), apatite (up to $10 \mathrm{~cm}, 1$ to $10 \mathrm{vol} . \%$ ), and large calcite grains $(1-3 \mathrm{~cm}$, up to 70 vol.\%). Apatites are pink and inclusionfree but can contain local monazite-calcite-allanite inclusions. We note the presence of two pegmatitic samples (IC2-21 and INH604) with yellow apatite and britholite inclusions. Only one 

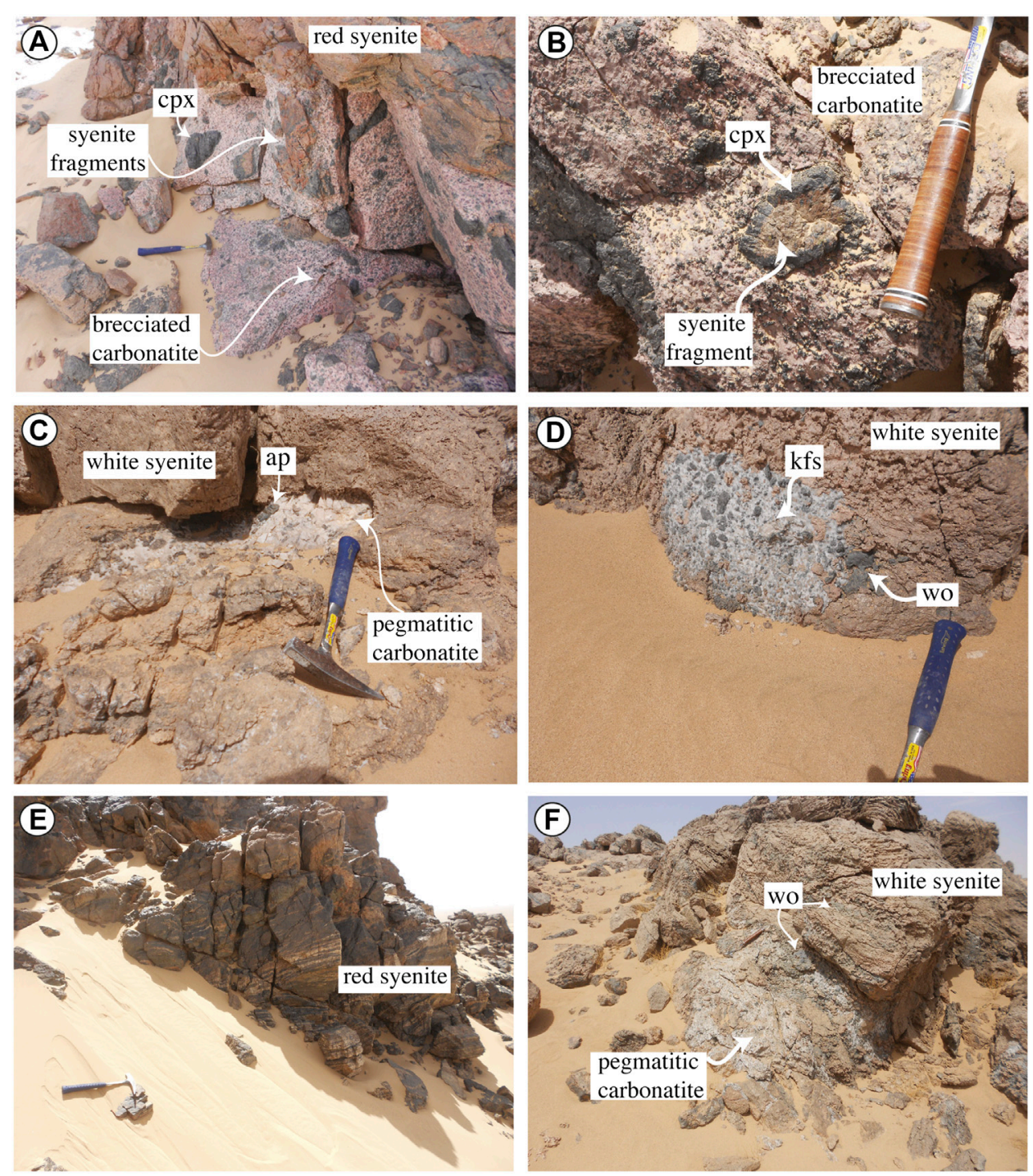

FIGURE 2| Field photographs of the alkaline syenites and carbonatites in the three sectors of lhouhaouene area. (A) North wadi Tirahart brecciated carbonatite vein with clinopyroxene (cpx) and syenite fragments intruding a red syenite. (B) Oued lhouhaouene syenite fragment surrounded by clinopyroxene aureole in brecciated carbonatite. (C) South wadi Tirahart pegmatitic carbonatite with apatite (ap) crystals in white syenite. (D) South wadi Tirahart carbonatite pocket rich in wollastonite (wo) and K-feldspar (kfs) in white syenite. (E) North wadi Tirahart layered red syenite with light K-feldspar levels and dark clinopyroxene levels. (F) South wadi Tirahart pegmatitic carbonatite vein intruding a white syenite rich in wollastonite levels.

Si-poor sample (INH604) has apatite associated with wollastonite (1 vol.\%) and alkali feldspar surrounded by garnet. Fluorite and quartz are present as interstitial accessory minerals or in apatite cracks $(<1$ vol.\%), and quartz can be present as inclusion in clinopyroxene. Magnetite is present associated with clinopyroxene $(<1$ vol.\%). Allanite has euhedral form in the calcite groundmass $(<1$ vol.\%) or mostly roundish shape as small minerals around apatite and clinopyroxene. Sparse titanite and alkali feldspar are observed in some carbonatites as isolated minerals in the calcite groundmass $(<0.5$ vol.\%).

Si-rich carbonatites (Figure 3B) contain clinopyroxene (>3 mm; 10-25 vol.\%), wollastonite ( $8 \mathrm{~mm} ;<10$ vol.\%), and green or yellow apatite (3-5 mm; 1 to 22 vol.\%) set in matrix of calcite (3-8 mm, 50 to 70 vol.\%). Green apatites are free of inclusions and differ from yellow apatites having britholite exsolutions (up to $40 \mathrm{vol} . \%$ ). Accessory minerals are allanite appearing as small roundish minerals (0.five to one vol.\%) around clinopyroxene or in apatite cracks, titanite ( $<0.5$ vol.\%), K-feldspars (4-8 mm, $<1$ vol.\%), interstitial quartz, fluorite $(<1$ vol.\%), and garnet $(<0.5$ vol.\%). Titanite $(<1 \mathrm{~mm})$ is present as isolated crystals in the calcite groundmass. K-feldspar is present as euhedral mineral (Supplementary Figures S1, and S2) and sometimes surrounded by coronitic garnet in the britholite rich samples. Corona texture of calcite associated with quartz around wollastonite represents late subsolidus reaction involving $\mathrm{CO}_{2}$-rich fluid (Figure 3B; Malvoisin et al., 2012). 

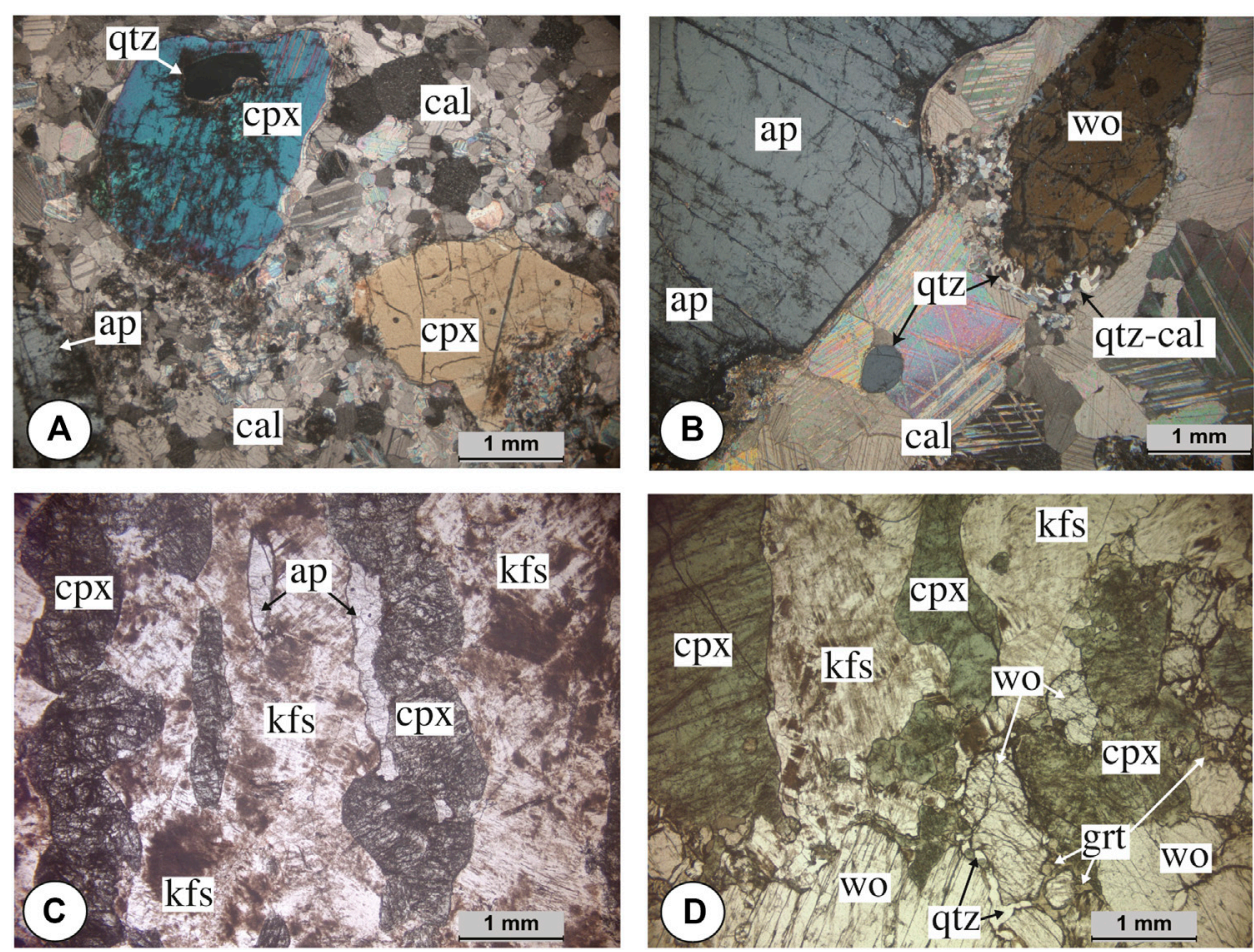

FIGURE 3| Microphotographs of (A-B) carbonatites and (C-D) syenites. (A) Si-poor brecciated carbonatite with calcite (cal), apatite (ap), and clinopyroxene (cpx) with quartz (qtz) inclusion. (B) Pegmatitic Si-rich carbonatite with apatite and wollastonite (wo) surrounded by quartz (qtz) and calcite. (C) Red syenite with altered K-feldspar (kfs) with apatite and clinopyroxene. (D) White syenite with K-feldspar, clinopyroxene, and wollastonite surrounded by garnet (grt) and quartz.

\section{Syenites}

Red syenites have medium- to coarse-grained magmatic-layered textures $(5-10 \mathrm{~mm}$, Figure 3C) with K-feldspar (3-9 mm, 40 to $80 \mathrm{vol} . \%$ ) with perthitic exsolution, clinopyroxene (3-8 mm, 20 to 40 vol.\%), and inclusion-free pink apatite (1-4 mm, 1 to 3 vol.\%) or inclusion-bearing pink apatite that contain local monazite-calcite-allanite inclusions and interstitial xenomorph quartz grains (1-2 vol.\%). Titanite with euhedral texture is present as accessory mineral ( $<2$ vol.\%) and associated with clinopyroxene. Allanite is present around clinopyroxene and magnetite as inclusions in clinopyroxene.

White syenites (Figure 3D) are present as magmatic-layered textures with light bands of K-feldspar, wollastonite $(5-10 \mathrm{~mm}$, 5-20\%), and yellow apatite with britholite and dark bands with clinopyroxene (3-8 mm, $40 \mathrm{vol} . \%)$. Garnet and magnetite are present as accessory minerals $(<0.5 \mathrm{vol} . \%)$. Garnet appears in an interstitial texture between clinopyroxenes or wollastonites and is associated with quartz. Titanite ( $<0.5 \mathrm{vol} . \%)$ is observed in the K-feldspar matrix.

\section{ANALYTICAL METHODS}

Fifty-four intrusive silicate and carbonatite rocks representing the diversity of rock types in the Ihouhaouene area were selected for petrological and geochemical study. Samples were chosen according to the localities and the different types of carbonatites (brecciated, pegmatitic, or calcite veins) and the two types of red and white syenites. Brecciated carbonatites were well separated and cleaned off syenite fragments before milling. A significant amount $(\sim 3 \mathrm{~kg})$ of pegmatitic carbonatites was milled to have a representative bulk-rock composition.

\section{Whole-Rock Major, Trace, and Volatile Element Analysis}

All samples were cut, crushed, and milled in agate into fine powders $(\geq 5 \mu \mathrm{m})$ without using water. Major elements were analyzed by ICP-OES (iCap ThermoFisher) at the SARM service of the CRPG (Nancy, France). The procedure followed is according to the protocol of Carignan et al. (2001). The wholerock powder was fused with $\mathrm{LiBO}_{2}$ and then dissolved in a $\mathrm{HNO}_{3}$, $\mathrm{H}_{2} \mathrm{O}$, and glycerol mixture. The relative standard deviation for the major elements is less than 5\%. Carbon and sulfur contents of the samples were determined using an element analyzer at the SARM service, whereas $\mathrm{F}$ and $\mathrm{Cl}$ were determined by wet precipitation ferrithiocyanate spectrophotometry using a Varian Cary 50 spectrophotometer.

Trace elements were analyzed using a quadrupole 7,700x ICPMass Spectrometry at Geosciences Montpellier (AETE platform OSU-OREME). An acid attack on $0.1 \mathrm{~g}$ of sample powder weighed in teflon-lined vessel is carried out with $1 \mathrm{ml}$ of $\mathrm{HClO}_{4}$ and $2.5 \mathrm{ml}$ of $\mathrm{HF}$ on a hot plate at $100^{\circ} \mathrm{C}$ for $24 \mathrm{~h}$. Hydrofluoric acid breaks the silicate link of rocks and puts the 
released ions in solution, while perchloric acid acts as a powerful oxidant. Then, the samples were evaporated to dryness at $150^{\circ} \mathrm{C}$. A second attack is applied with $0.5 \mathrm{ml}$ of $\mathrm{HClO}_{4}$ and $1 \mathrm{ml}$ of $\mathrm{HF}$ for $24 \mathrm{~h}$ followed by evaporation, and the samples are then taken up in three steps, separated by evaporation, in $0.5 \mathrm{ml}, 0.25 \mathrm{ml}$, and then $0.25 \mathrm{ml}$ of $\mathrm{HClO}_{4}$. The sample is evaporated to a solid residue. The first dilution is accomplished with this residue by adding nitric acid and MilliQ water until having a solution of $20.6 \mathrm{~g}$. Before mass spectrometer analysis, the sample solution is diluted with $9.45 \mathrm{ml}$ of $\mathrm{H}_{2} \mathrm{O}, 0.25 \mathrm{ml}$ of $\mathrm{HNO}_{3}$, and 0.1 of $\mathrm{InBi}$ according to a factor of 10,000 , which has been chosen for the associated carbonatites and syenites. For each series of analysis (20 samples), four blanks (only acids were introduced into Savilex teflon-lined vessel) and four standards, BHVO-1, BEN, UBN, and COQ-1, were analyzed to monitor internal drift. The mass spectrometer used to quantify trace element concentrations in whole rock is Agilent 7,700x MC-ICPMS. The sensitivity is of the order of $200 \times 106 \mathrm{cps} / \mathrm{ppm}$ on ${ }^{115} \mathrm{In}$ (indium). Internal standards $\mathrm{A} 2$ and $\mathrm{A} 4$ are calibrated for $\mathrm{Zr}-\mathrm{Nb}$ and $\mathrm{Hf}-\mathrm{Ta}$ concentrations, and calibration curves on $\mathrm{Rb}, \mathrm{Sr}, \mathrm{Y}, \mathrm{Zr}$, and $\mathrm{Ce}$ are obtained to verify the internal calibration.

\section{In Situ Mineral Analysis for Major, Trace, and Volatile Elements}

Major and volatile element concentrations in minerals were analyzed using electron microprobe (CAMECA SX-100 at Geosciences Montpellier, France). Peaksight software was used to perform calibration, overlap correction, and quantification. Raw data processing for matrix corrections was performed using ZAF and PAP models (Castaing, 1963). Acceleration voltage and beam current were set to $20 \mathrm{kV}$ and $10 \mathrm{nA}$, and a focused beam $(1 \mu \mathrm{m})$ was used except for carbonates that were analyzed with a defocused beam $(10 \mu \mathrm{m})$. The background counting time was fixed at $20 \mathrm{~s}$ for major elements and $40 \mathrm{~s}$ for $\mathrm{F}, \mathrm{Cl}$, and $\mathrm{S}$. The analyses were calibrated on reference material standards chosen for each element: $\mathrm{Al}_{2} \mathrm{O}_{3}$ for $\mathrm{Al}, \mathrm{TiO}_{2}$ for $\mathrm{Ti}$, wollastonite for $\mathrm{Ca}$ and $\mathrm{Si}$, albite for $\mathrm{Na}$, orthoclase for $\mathrm{K}$, forsterite for $\mathrm{Mg}$, hematite for $\mathrm{Fe}$, apatite for $\mathrm{P}$, native metal for $\mathrm{Ni}, \mathrm{Mn}$, and $\mathrm{Cu}$, celestine for $\mathrm{Sr}, \mathrm{ThO}_{2}$ for Th, glasses for REE, baryte for $\mathrm{S}$ and $\mathrm{Ba}$, fluorite for $\mathrm{F}$, and chloroapatite for $\mathrm{Cl}$. We observed $\mathrm{F}$ migration in apatite for the analytical conditions used for major and minor element analyses (Goldoff et al., 2012). The F content has been estimated using reference apatite crystal oriented with its c-axis perpendicular to the incident electron beam with $3.4 \mathrm{wt}$.\% F.

Trace element analyses of minerals were determined by LAHR-ICP-MS at Geosciences Montpellier (AETE platform, OSUOREME), using GeoLas Q + Excimer CompEx102. A laser beam of diameter $26 \mu \mathrm{m}$ was used for apatite and britholite, $51 \mu \mathrm{m}$ for silicate minerals, and $77 \mu \mathrm{m}$ for calcite and wollastonite with a repetition rate of $7 \mathrm{~Hz}$, a laser power of $5 \mathrm{~J} / \mathrm{cm}^{2}$, and a mixture of helium and argon as carrier gas. Each analysis consisted of a preablation pulse of $10 \mathrm{~s}, 2$ min measurement of the background, and $60 \mathrm{~s}$ integration while the laser fires. The concentrations were calibrated with NIST 612 and the $\mathrm{SiO}_{2}-\mathrm{CaO}$ concentrations determined previously with electron microprobe for each mineral. BIR-G1 standard is used as external standard. The detection limits depended on the day-to-day tuning of the ICPMS, the "gas blank" data obtained from the mass spectrometer before the laser is turned (background), the laser spot size, and the element being analyzed. Two-minute measurement of the background was performed for optimization of the peak integration. The instrument sensitivity due to analytical conditions was determined from the average across all days of repeat measurements of the synthetic NIST 612 glass (Pearce et al., 1997). Data were processed with Glitter software (Griffin et al., 2008), and elements were considered below detection and filtered out of the dataset if the concentration had a standard deviation (1 sigma) that was larger than the value of the detection limit. Values for standards and detection limit are reported in Supplementary Table S2.

\section{WHOLE-ROCK COMPOSITION}

\section{Carbonatites}

All carbonatites are calciocarbonatites with $\mathrm{CaO} /(\mathrm{CaO}+\mathrm{MgO}+$ $\mathrm{FeOt}+\mathrm{MnO}$ ) $>0.8$ (Gittins and Harmer, 1997). The silica and calcium contents in carbonatites range from 3 to $35 \mathrm{wt} . \%$ and 28.70 to 53.02 wt.\%, respectively (Table 1). According to the silicate mineral proportions with brecciated and pegmatitic textures (in both massive and vein outcrops, see above) and the classification from Woolley and Kempe (1989), we distinguish two groups of carbonatites as a function of silica content: Si-poor carbonatites with $\mathrm{SiO}_{2}<20$ wt.\% and Si-rich carbonatites (or silicocarbonatites) with $\mathrm{SiO}_{2}>20$ wt.\%.

Si-poor carbonatites (Table 1) have high $\mathrm{CaO}$ and $\mathrm{CO}_{2}$ content (42.18-53.02 wt.\% and 24.83 to 36.15 wt.\%, respectively), decreasing with increasing $\mathrm{SiO}_{2}$ content, variable FeO (0.37-4.10 wt.\%), $\mathrm{MgO}(0.11-3.21$ wt.\%, $\mathrm{Mg} \#=0.54-0.84)$, $\mathrm{Al}_{2} \mathrm{O}_{3}\left(0.11-2.82\right.$ wt.\%), and $\mathrm{P}_{2} \mathrm{O}_{5}$ (0.79-5.86 wt.\%), and very low $\mathrm{Na}_{2} \mathrm{O}$ and $\mathrm{K}_{2} \mathrm{O}$ contents $\left(\mathrm{Na}_{2} \mathrm{O}+\mathrm{K}_{2} \mathrm{O}=0.15\right.$ to 1.39 wt.\%) (Figures 4, 5). Volatile content ranges from 53 to $210 \mathrm{ppm} \mathrm{Cl}$, 0.06 to 0.44 wt. $\% \mathrm{~F}$, and 0.01 to 0.11 wt.\% S.

Si-rich carbonatites have lower $\mathrm{CaO}$ (28.69-42.88 wt.\%) and $\mathrm{CO}_{2}$ (11.34-24.56 wt.\%) contents and higher $\mathrm{Al}_{2} \mathrm{O}_{3}$ (2.01-8.14 wt.\%) content than Si-poor carbonatites that decrease linearly with increasing $\mathrm{SiO}_{2}$ content. The concentrations are variable for $\mathrm{P}_{2} \mathrm{O}_{5}$ (0.71-4.02 wt.\%), $\mathrm{MgO}$ (0.58-3.31 wt.\%, $\mathrm{Mg \#}=0.55-0.76)$, and $\mathrm{FeO}(1.09-4.09$ wt.\%). The alkali contents increase from 1.28 to 6.20 wt. $\% \mathrm{Na}_{2} \mathrm{O}+\mathrm{K}_{2} \mathrm{O}$ (Figures 4, 5), and volatile contents range from 52 to $195 \mathrm{ppm} \mathrm{Cl}$, 0.07 to 0.62 wt.\% F, and 0.03 to 0.13 wt.\% S (Table 1).

Carbonatites have high trace element concentrations (Table 1; Figure 6). Si-poor carbonatites have straight REE patterns, enriched in 1,253 to 8,816 times chondrite. They are enriched in light rare earth elements (LREE) compared to heavy rare earth elements (HREE) on a chondrite-normalized plot (excluding INH 604 sample that is essentially composed of apatite) with negative anomaly in $\mathrm{Eu}\left(\mathrm{Eu} / \mathrm{Eu}^{*}=0.73\right.$ to $0.8 ; \mathrm{La}_{\mathrm{N}} /$ $\mathrm{Yb}_{\mathrm{N}}=75-203$; Figure 6A). The high REE concentrations recorded in $\mathrm{Si}$-poor carbonatites could be related to the high apatite modal proportion. Relative to the primitive mantle 
TABLE 1 | Representative major and trace element whole-rock compositions of carbonatites and syenites.

\begin{tabular}{|c|c|c|c|c|c|c|c|c|c|c|c|c|c|c|c|c|c|}
\hline \multirow{2}{*}{$\frac{\text { Rock type }}{\text { Sample }}$} & \multicolumn{14}{|c|}{ Si-poor carbonatites } & \multicolumn{3}{|c|}{ Si-rich carbonatites } \\
\hline & IC3-21 & IC1-4C & IC3-7 & IC2-21 & INH642 & IC3-17 & IC3-20 & INH604 & IC3-1 & IC3-2 & IC1-12B & IC1-4 & IC3-15 & IC3-5 & IC2-20 & IC2-9 & IC3-19 \\
\hline $\mathrm{SiO}_{2}$ (wt.\%) & 2.7 & 3.94 & 4.56 & 5.42 & 5.52 & 7.88 & 8.63 & 8.81 & 9.63 & 10.11 & 10.47 & 10.82 & 17.18 & 17.68 & 21.58 & 21.91 & 25.62 \\
\hline $\mathrm{TiO}_{2}$ & 0.02 & - & 0.03 & - & 0.04 & 0.07 & 0.06 & 0.04 & 0.07 & 0.04 & 0.06 & 0.07 & 0.12 & 0.15 & 0.09 & 0.63 & 0.12 \\
\hline $\mathrm{Al}_{2} \mathrm{O}_{3}$ & 0.58 & 0.47 & 0.81 & 0.11 & 0.67 & 1.26 & 1.23 & 0.16 & 2.03 & 1.78 & 1.67 & 2.1 & 2.82 & 2.52 & 4.46 & 3.33 & 4.64 \\
\hline $\mathrm{Fe}_{2} \mathrm{O}_{3}$ & 0.5 & 0.89 & 1.08 & 0.85 & 1.32 & 3.84 & 2.51 & 0.37 & 4.1 & 0.89 & 1.18 & 2.41 & 2.3 & 3.67 & 2.08 & 1.84 & 2.36 \\
\hline $\mathrm{MnO}$ & 0.08 & 0.19 & 0.17 & 0.14 & 0.14 & 0.19 & 0.16 & 0.1 & 0.17 & 0.16 & 0.17 & 0.16 & 0.17 & 0.2 & 0.22 & 0.17 & 0.2 \\
\hline $\mathrm{MgO}$ & 0.26 & 0.87 & 1.05 & 1.01 & 1.06 & 1.36 & 1.52 & 0.11 & 2.11 & 1.19 & 1.19 & 1.74 & 3.12 & 3.21 & 1.54 & 0.58 & 1.8 \\
\hline $\mathrm{CaO}$ & 53.02 & 51.87 & 50.97 & 50.63 & 50.62 & 48.57 & 49.03 & 51 & 45.33 & 47.42 & 46.63 & 47 & 42.31 & 42.18 & 37.16 & 41.18 & 35.78 \\
\hline $\mathrm{Na}_{2} \mathrm{O}$ & 0.05 & 0.03 & 0.08 & 0.03 & 0.05 & 0.12 & 0.1 & - & 0.08 & 0.13 & 0.15 & 0.27 & 0.33 & 0.26 & 0.38 & 0.16 & 0.6 \\
\hline $\mathrm{K}_{2} \mathrm{O}$ & 0.2 & 0.07 & 0.12 & - & 0.07 & 0.04 & 0.1 & _- & 0.07 & 1.12 & 1 & 0.26 & 1.06 & 0.57 & 3.03 & 2.63 & 2.8 \\
\hline $\mathrm{P}_{2} \mathrm{O}_{5}$ & 1.07 & 2.39 & 5.47 & 0.79 & 5.86 & 4.02 & 3.44 & 5.62 & 3.48 & 0.81 & 1.24 & 3.2 & 2.01 & 2.15 & 0.98 & 2.12 & 1.35 \\
\hline LOI & 40.59 & 37.96 & 33.86 & 38.75 & 33.31 & 31.31 & 32.21 & 29.61 & 31.51 & 35.26 & 34.87 & 30.6 & 27.23 & 26.58 & 26.39 & 24.44 & 23.54 \\
\hline Total & 99.08 & 98.66 & 98.19 & 97.71 & 98.65 & 98.64 & 98.98 & 95.8 & 98.57 & 98.91 & 98.62 & 98.62 & 98.65 & 99.17 & 97.89 & 98.97 & 98.8 \\
\hline $\mathrm{CO}_{2}$ & 36.15 & 34.38 & 30.10 & 36.02 & 30 & 27.15 & 29.97 & 28.05 & 29.75 & 32.42 & 31.73 & 26.08 & 26.08 & 24.83 & 24.56 & 22.65 & 20.64 \\
\hline $\mathrm{F}$ & 0.08 & 0.2 & 0.39 & 0.06 & 0.44 & 0.3 & 0.24 & 0.37 & 0.26 & 0.06 & 0.11 & 0.24 & 0.16 & 0.18 & 0.07 & 0.19 & 0.12 \\
\hline $\mathrm{S}$ & 0.01 & $<0.01$ & 0.03 & 0.11 & 0.03 & 0.01 & $<0.01$ & 0.08 & 0.04 & $<0.01$ & $<0.01$ & $<0.01$ & $<0.01$ & 0.01 & 0.13 & 0.03 & $<0.01$ \\
\hline $\mathrm{Cl}(\mathrm{ppm})$ & 105 & 130 & 88 & 120 & 105 & 120 & 64 & 210 & 110 & 95 & 53 & 76 & 88 & 71 & 52 & 140 & 87 \\
\hline Mg\# & 0.68 & 0.79 & 0.79 & 0.82 & 0.76 & 0.59 & 0.71 & 0.54 & 0.67 & 0.84 & 0.8 & 0.74 & 0.84 & 0.78 & 0.75 & 0.55 & 0.75 \\
\hline$(\mathrm{N}+\mathrm{K})$ ox/Alox & 0.44 & 0.2 & 0.25 & - & 0.17 & 0.12 & 0.16 & - & 0.07 & 0.7 & 0.69 & 0.25 & 0.49 & 0.33 & 0.76 & 0.84 & 0.73 \\
\hline Cs (ppm) & 0.02 & 0.02 & 0.04 & 0.01 & 0.01 & 0.01 & 0.01 & 0.01 & 0.02 & 0.24 & 0.3 & 0.02 & 0.26 & 0.08 & 0.9 & 0.49 & 0.69 \\
\hline $\mathrm{Rb}$ & 5.94 & 1.73 & 6.16 & 0.15 & 2.88 & 0.9 & 2.86 & 0.55 & 0.53 & 30.78 & 28.43 & 5.86 & 33.66 & 16.24 & 84.4 & 57.72 & 88.18 \\
\hline $\mathrm{Ba}$ & 270 & 71 & 120 & 672 & 264 & 279 & 215 & 1,221 & 290 & 745 & 931 & 229 & 995 & 606 & 3,104 & 1,364 & 1868 \\
\hline Th & 32.85 & 36.26 & 187.16 & 17.92 & 174 & 36 & 40 & 493 & 7.77 & 68.83 & 75.67 & 22.91 & 69.52 & 62.28 & 29 & 201 & 114 \\
\hline U & 2.91 & 4.81 & 10.59 & 11.11 & 9.7 & 1.22 & 3.63 & 74.62 & 0.1 & 6.14 & 9.08 & 2.1 & 3.59 & 4.57 & 14.67 & 34.28 & 32.7 \\
\hline $\mathrm{Nb}$ & 3.4 & 0.79 & 0.53 & 0.73 & 0.9 & 0.65 & 0.42 & 6.57 & 0.04 & 4 & 5.94 & 0.39 & 1.87 & 2.18 & 32.27 & 60.09 & 25.75 \\
\hline $\mathrm{Ta}$ & 0.39 & 0.05 & 0.07 & 0.46 & 0.33 & 0.13 & 0.12 & 1.3 & 0.08 & 0.76 & 1.13 & 0.06 & 0.25 & 0.22 & 9.51 & 11.95 & 3.83 \\
\hline $\mathrm{La}$ & 1,130 & 655 & 1,377 & 627 & 2089 & 941 & 1,014 & 3,956 & 297 & 1845 & 1,216 & 878 & 1,357 & 1,050 & 624 & 1,372 & 891 \\
\hline $\mathrm{Ce}$ & 1988 & 1,348 & 2,715 & 1,196 & 4,006 & 1825 & 1958 & 8,185 & 585 & 3,275 & 2,213 & 1,650 & 2,603 & 1939 & 1,196 & 3,111 & 1881 \\
\hline $\mathrm{Pb}$ & 66 & 59 & 70 & 1933 & 73 & 39 & 16 & 129 & 18 & 54 & 259 & 88 & 47 & 84 & 1,436 & 54 & 302 \\
\hline Pr & 216 & 166 & 322 & 134 & 454 & 212 & 227 & 845 & 70 & 353 & 243 & 189 & 296 & 216 & 134 & 332 & 200 \\
\hline $\mathrm{Sr}$ & 3,705 & 6,259 & 5,451 & 11,446 & 5,204 & 4,329 & 4,751 & 3,664 & 1969 & 4,091 & 6,786 & 3,124 & 5,342 & 3,729 & 8,546 & 3,397 & 3,481 \\
\hline $\mathrm{Nd}$ & 779 & 657 & 1,218 & 487 & 1,679 & 805 & 859 & 3,037 & 270 & 1,227 & 867 & 710 & 1,091 & 788 & 480 & 1,228 & 734 \\
\hline $\mathrm{Zr}$ & 8.43 & 9.52 & 9.12 & 13.79 & 11.37 & 15.89 & 29.32 & 59.7 & 19.29 & 25.17 & 26.15 & 14 & 66.45 & 46.13 & 34.22 & 205 & 66.4 \\
\hline $\mathrm{Hf}$ & 0.21 & 0.32 & 0.2 & 0.29 & 0.33 & 0.37 & 0.74 & 0.51 & 0.35 & 0.9 & 1.27 & 0.38 & 2.08 & 1.68 & 0.96 & 4.34 & 1.84 \\
\hline $\mathrm{Sm}$ & 112 & 115 & 196 & 74 & 256 & 131 & 136 & 444 & 44 & 172 & 127 & 113 & 165 & 121 & 72 & 190 & 115 \\
\hline Eu & 22.06 & 25.07 & 38.82 & 14.9 & 49.62 & 27.51 & 28.43 & 86.54 & 9.49 & 34.54 & 25.09 & 23.8 & 33.28 & 24.08 & 14.49 & 39.58 & 22.72 \\
\hline Gd & 71.63 & 81.22 & 126 & 47.62 & 168 & 87.22 & 91.13 & 281 & 29.21 & 111 & 82 & 75 & 101 & 77.11 & 44.42 & 115 & 72 \\
\hline $\mathrm{Tb}$ & 7.42 & 8.63 & 13.09 & 5.17 & 16.75 & 9.04 & 9.44 & 26.56 & 3.04 & 10.58 & 8.12 & 7.92 & 10.22 & 8.33 & 4.71 & 12.1 & 7.67 \\
\hline Dy & 31.16 & 37 & 55 & 22.44 & 68.36 & 37.7 & 39.67 & 104 & 12.61 & 41.32 & 32.72 & 33.47 & 41.3 & 35.7 & 19.7 & 49.34 & 32.02 \\
\hline Y & 125 & 144 & 208 & 95 & 248 & 146 & 150 & 369 & 46 & 163 & 121 & 127 & 149 & 137 & 73 & 184 & 117 \\
\hline $\mathrm{Ho}$ & 4.62 & 5.39 & 7.96 & 3.39 & 9.66 & 5.4 & 5.75 & 14.06 & 1.8 & 5.74 & 4.64 & 4.88 & 5.75 & 5.32 & 2.86 & 6.89 & 4.6 \\
\hline Er & 9.44 & 10.75 & 16.04 & 7.3 & 19.34 & 10.81 & 11.47 & 27.32 & 3.54 & 11.5 & 9.49 & 9.78 & 11.22 & 11.07 & 5.75 & 13.55 & 9.17 \\
\hline $\mathrm{Tm}$ & 1.08 & 1.2 & 1.78 & 0.88 & 2.08 & 1.19 & 1.26 & 2.83 & 0.38 & 1.27 & 1.07 & 1.09 & 1.2 & 1.26 & 0.65 & 1.49 & 1.03 \\
\hline $\mathrm{Yb}$ & 5.61 & 6.23 & 9.06 & 4.98 & 10.31 & 6.11 & 6.48 & 13.45 & 1.94 & 6.51 & 5.48 & 5.72 & 6.18 & 6.81 & 3.48 & 7.57 & 5.45 \\
\hline Lu & 0.7 & 0.8 & 1.09 & 0.64 & 1.25 & 0.78 & 0.82 & 1.57 & 0.24 & 0.82 & 0.7 & 0.73 & 0.75 & 0.88 & 0.46 & 0.92 & 0.7 \\
\hline $\mathrm{Eu} / \mathrm{Eu}^{\star}$ & 0.75 & 0.79 & 0.76 & 0.77 & 0.73 & 0.79 & 0.78 & 0.75 & 0.81 & 0.76 & 0.75 & 0.79 & 0.79 & 0.76 & 0.79 & 0.82 & 0.76 \\
\hline
\end{tabular}


TABLE 1 | (Continued) Representative major and trace element whole-rock compositions of carbonatites and syenites.

\begin{tabular}{|c|c|c|c|c|c|c|c|c|c|c|c|c|c|c|c|c|}
\hline \multirow{2}{*}{$\begin{array}{l}\text { Rock type } \\
\text { Sample }\end{array}$} & \multicolumn{13}{|c|}{ Si-poor carbonatites } & \multicolumn{3}{|c|}{ Si-rich carbonatites } \\
\hline & IC3-21 & IC1-4C & IC3-7 & IC2-21 & INH642 & IC3-17 & IC3-20 & INH604 & IC3-1 & IC3-2 & IC1-12B & IC1-4 & IC3-5 & IC2-20 & IC2-9 & IC3-19 \\
\hline $\mathrm{Nb} / \mathrm{Ta}$ & 8.63 & 17.35 & 7.88 & 1.6 & 2.71 & 4.97 & 3.38 & 5.04 & 0.56 & 5.25 & 5.27 & 6.79 & 9.76 & 3.39 & 5.03 & 6.73 \\
\hline $\mathrm{Zr} / \mathrm{Hf}$ & 40.68 & 29.73 & 45.64 & 48.24 & 34.33 & 42.72 & 39.7 & 116.3 & 55.77 & 28.03 & 20.52 & 36.93 & 27.5 & 35.56 & 47.2 & 36.14 \\
\hline Rock type & \multicolumn{4}{|c|}{ Si-rich carbonatites } & \multicolumn{6}{|c|}{ Red syenites } & \multicolumn{6}{|c|}{ White syenites } \\
\hline Sample & IC1-12 & IC2-11 & IHN170 & IC1-2 & IC1-13 & IC1-12A & IC3-4 & IC1-24A & IC1-24B & IC2-6A & IC2-27 & IC3-18 & IC2-11S & IC3-10 & IC3-12B & IC2-10 \\
\hline $\mathrm{SiO}_{2}$ (wt.\%) & 26.52 & 27.88 & 33.38 & 35.1 & 57.49 & 60.91 & 61.36 & 64.13 & 65.21 & 71.67 & 52.08 & 52.66 & 52.75 & 55.26 & 57.83 & 58.47 \\
\hline $\mathrm{TiO}_{2}$ & 0.06 & 0.19 & 0.06 & 0.06 & 0.17 & 0.32 & 0.03 & 0.55 & 0.38 & 0.22 & 0.16 & 2.05 & 0.09 & 0.05 & 0.21 & 0.03 \\
\hline $\mathrm{Al}_{2} \mathrm{O}_{3}$ & 2.2 & 6.53 & 2.01 & 8.14 & 8.61 & 15.28 & 14.59 & 16.09 & 15.14 & 13.6 & 3.35 & 13.88 & 14.97 & 18.87 & 13.02 & 12.89 \\
\hline $\mathrm{Fe}_{2} \mathrm{O}_{3}$ & 4.09 & 2.04 & 2 & 1.09 & 4.22 & 3.32 & 1.82 & 2.4 & 2.02 & 1.76 & 4.41 & 7.01 & 0.69 & 4.53 & 2.16 & 1.25 \\
\hline $\mathrm{MnO}$ & 0.49 & 0.12 & 0.31 & 0.1 & 0.43 & 0.17 & 0.14 & 0.06 & 0.09 & 0.09 & 0.57 & 0.16 & 0.03 & 0.08 & 0.25 & 0.2 \\
\hline $\mathrm{MgO}$ & 3.31 & 0.77 & 1.22 & 0.69 & 7.68 & 1.57 & 3.34 & 0.77 & 0.68 & 0.53 & 1.31 & 1.65 & 0.18 & 2.99 & 0.81 & 0.22 \\
\hline $\mathrm{CaO}$ & 37.46 & 32.42 & 42.88 & 28.7 & 12.78 & 5.11 & 5.33 & 2.56 & 2.74 & 1.8 & 33.87 & 14.26 & 10.14 & 6.89 & 13.19 & 13.7 \\
\hline $\mathrm{Na}_{2} \mathrm{O}$ & 0.24 & 0.46 & 0.25 & 0.88 & 1.33 & 2.07 & 1.21 & 2.87 & 1.64 & 2.89 & 0.24 & 1.95 & 0.93 & 5.42 & 1.59 & 1.01 \\
\hline $\mathrm{K}_{2} \mathrm{O}$ & 1.3 & 5.04 & 1.04 & 5.32 & 5.23 & 9.97 & 11.2 & 9.41 & 10.68 & 7.19 & 2.41 & 2.29 & 11.84 & 1.77 & 7.62 & 9.69 \\
\hline $\mathrm{P}_{2} \mathrm{O}_{5}$ & 0.71 & 1.64 & 4.02 & 1.61 & 0.43 & 0.48 & 0.55 & 0.25 & 0.12 & 0.21 & 0.29 & 0.43 & 0.45 & 2.03 & 0.72 & 0.76 \\
\hline LOI & 24.38 & 22.72 & 10.91 & 17.15 & 1.02 & 1.04 & 0.62 & 0.87 & 1.07 & 0.92 & 1.13 & 3.28 & 7.41 & 2.16 & 2.53 & 1.60 \\
\hline Total & 100.74 & 99.8 & 98.07 & 98.82 & 99.37 & 100.24 & 100.19 & 99.94 & 99.76 & 100.87 & 99.82 & 99.6 & 99.47 & 100.04 & 99.91 & 99.83 \\
\hline $\mathrm{CO}_{2}$ & 21.83 & 20.41 & 11.34 & 15.91 & 0.22 & 0.42 & 0.19 & 0.14 & 0.4 & 0.11 & 0.91 & 2.02 & 6.49 & 0.18 & 1.42 & 1.19 \\
\hline $\mathrm{F}$ & 0.07 & 0.15 & 0.62 & 0.37 & 0.1 & 0.05 & 0.07 & 0.05 & 0.03 & 0.03 & 0.03 & 0.1 & 0.04 & 0.19 & 0.07 & 0.06 \\
\hline S & $<0.01$ & 0.03 & 0.13 & 0.06 & 0.03 & $<0.01$ & $<0.01$ & $<0.01$ & 0.02 & $<0.01$ & 0.01 & 0.09 & 0.01 & $<0.01$ & 0.04 & 0.02 \\
\hline $\mathrm{Cl}(\mathrm{ppm})$ & 125 & 100 & 135 & 195 & 83 & 135 & 115 & 78 & 72 & 50 & 36 & 570 & 37 & 210 & 115 & 45 \\
\hline Mg\# & 0.76 & 0.6 & 0.71 & 0.71 & 0.88 & 0.65 & 0.88 & 0.56 & 0.57 & 0.54 & 0.54 & 0.48 & 0.51 & 0.72 & 0.6 & 0.41 \\
\hline$(\mathrm{N}+\mathrm{K})$ ox/Alox & 0.7 & 0.84 & 0.64 & 0.76 & 0.79 & 0.76 & 0.85 & 0.76 & 0.81 & 0.74 & 0.79 & 0.3 & 0.85 & 0.38 & 0.71 & 0.83 \\
\hline Cs (ppm) & 0.31 & 1 & 0.24 & 1.46 & 0.48 & 1.82 & 2.82 & 0.55 & 0.75 & 0.22 & 0.55 & 0.9 & 2.71 & 0.09 & 2.05 & 1.48 \\
\hline $\mathrm{Rb}$ & 39.7 & 148.06 & 29.79 & 183 & 138 & 251 & 388 & 219 & 275 & 171 & 63.5 & 82.3 & 321 & 23.5 & 217 & 229 \\
\hline $\mathrm{Ba}$ & 898 & 2620 & 559 & 2505 & 698 & 4198 & 1297 & 2194 & 2940 & 2073 & 1316 & 1228 & 6015 & 900 & 13198 & 4760 \\
\hline Th & 93.77 & 112 & 154 & 85.89 & 32 & 48 & 16 & 9 & 63 & 80 & 32 & 23 & 20 & 5 & 109 & 62 \\
\hline U & 13.25 & 41.53 & 20.74 & 11.52 & 2.04 & 5.07 & 1.55 & 0.8 & 6.02 & 8.81 & 5.69 & 1.88 & 5.21 & 0.32 & 45.99 & 15.61 \\
\hline $\mathrm{Nb}$ & 6.05 & 18.75 & 3.96 & 11.15 & 10.54 & 16 & 0.73 & 64.61 & 48.04 & 26.7 & 3.96 & 86 & 7.82 & 0.12 & 61.26 & 1.59 \\
\hline $\mathrm{Ta}$ & 0.83 & 4.58 & 0.38 & 1 & 1.78 & 1.38 & 0.03 & 6.02 & 4.82 & 3.37 & 0.77 & 8 & 1.1 & 0.03 & 8.21 & 0.2 \\
\hline $\mathrm{La}$ & 780 & 1790 & 1379 & 857 & 261 & 206 & 226 & 136 & 242 & 219 & 175 & 231 & 385 & 207 & 441 & 323 \\
\hline $\mathrm{Ce}$ & 1480 & 3861 & 2719 & 1578 & 540 & 498 & 494 & 362 & 523 & 468 & 419 & 574 & 843 & 408 & 1070 & 821 \\
\hline $\mathrm{Pb}$ & 421 & 68 & 104 & 325 & 28 & 204 & 31 & 44 & 47 & 56 & 22 & 49 & 28 & 16 & 88 & 33 \\
\hline $\operatorname{Pr}$ & 167 & 399 & 309 & 173 & 66 & 65 & 63 & 53 & 63 & 56 & 50 & 77 & 89 & 48 & 108 & 94 \\
\hline $\mathrm{Sr}$ & 5105 & 3308 & 3541 & 5310 & 781 & 2313 & 744 & 982 & 553 & 793 & 433 & 1531 & 1571 & 1410 & 1595 & 871 \\
\hline $\mathrm{Nd}$ & 606 & 1461 & 1111 & 618 & 245 & 256 & 246 & 219 & 230 & 208 & 198 & 306 & 333 & 177 & 399 & 355 \\
\hline $\mathrm{Zr}$ & 22 & 81.28 & 23.05 & 78.74 & 56.24 & 45.86 & 6.8 & 22.76 & 22 & 197 & 24.68 & 160 & 22.36 & 1.21 & 58.93 & 13.03 \\
\hline $\mathrm{Hf}$ & 0.98 & 1.37 & 0.63 & 2.83 & 3.11 & 2.18 & 0.43 & 1.7 & 1.1 & 6.61 & 0.59 & 7.65 & 0.41 & 0.07 & 1.87 & 0.19 \\
\hline $\mathrm{Sm}$ & 92 & 217 & 170 & 93 & 39.7 & 42 & 42.18 & 41.74 & 35.9 & 34.62 & 36.67 & 51.77 & 51.14 & 27.18 & 65.85 & 57 \\
\hline Eu & 18.7 & 44.92 & 31.71 & 18.22 & 8.11 & 8 & 8.33 & 5.53 & 7 & 6.1 & 8.35 & 9.57 & 11.51 & 3.28 & 12.9 & 12.38 \\
\hline $\mathrm{Gd}$ & 56.62 & 130 & 115 & 59.3 & 26 & 26.7 & 28 & 30.82 & 22.24 & 23.37 & 28.23 & 35.37 & 30.25 & 19.27 & 46.03 & 34.77 \\
\hline $\mathrm{Tb}$ & 5.97 & 13.65 & 12.11 & 6.53 & 2.67 & 2.9 & 3 & 4.09 & 2.43 & 2.68 & 3.53 & 3.91 & 3.22 & 2.14 & 5 & 3.85 \\
\hline Dy & 24.8 & 55.62 & 51.81 & 29 & 11.06 & 12.42 & 12.43 & 21.2 & 10.44 & 12.2 & 17.7 & 17.76 & 13.37 & 9.87 & 21.8 & 16.71 \\
\hline Y & 91 & 207 & 217 & 117 & 40.55 & 43.13 & 44 & 83.02 & 38 & 50.33 & 84.15 & 74.6 & 49.39 & 38.17 & 84.42 & 72.37 \\
\hline
\end{tabular}




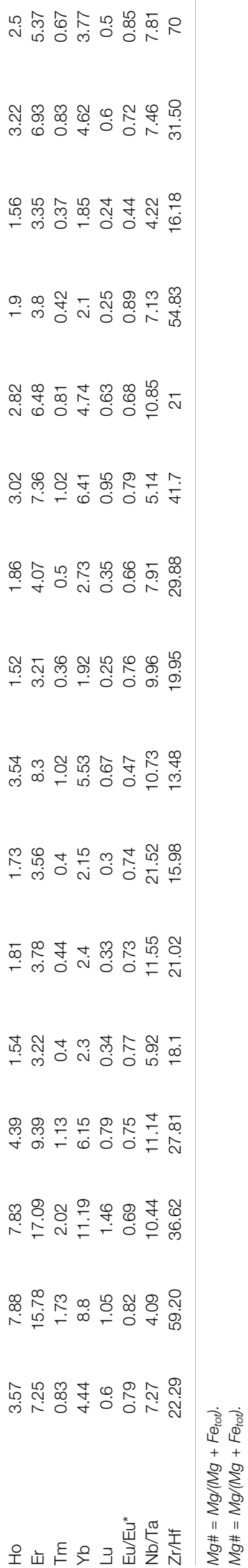

(PM), Si-poor carbonatites have low and variable concentrations of large-ion lithophile element (LILE: 0.01-0.30 ppm Cs, 0.90-33.66 ppm Rb, and 71.48-995 ppm $\mathrm{Ba}$, excluding INH 604 sample) with high content in Th (Table 1; Figure 6B). $\mathrm{Nb}$ is fractionated from $\mathrm{Ta}(\mathrm{Nb} / \mathrm{Ta}=$ 0.56-17.35) with high fractionation and negative anomaly in $\mathrm{Zr}-\mathrm{Hf}(\mathrm{Zr} / \mathrm{Hf}=20.52-55.77)$.

Si-rich carbonatite REE patterns are similar to those of Sipoor carbonatites. They are enriched in 2,633 to 7,551 times chondrite with a slight negative anomaly in $\mathrm{Eu}\left(\mathrm{Eu} / \mathrm{Eu}^{*}=0.69\right.$ to 0.82 ; Figure 6C) and $\mathrm{La}_{N} / \mathrm{Yb}_{\mathrm{N}}$ ratio from 88 to 145 . The concentrations of LILE and high-field-strength elements (HFSEs) in Si-rich carbonatites are higher than in Si-poor carbonatites (0.31-1.46 ppm Cs, 29.79-183 ppm Rb, 559-3,104 ppm Ba) with high contents in $\mathrm{U}$ and Th (Table 1; Figure 6D) and $\mathrm{Nb}-\mathrm{Ta}$ and $\mathrm{Zr}-\mathrm{Hf}$ fractionation $(\mathrm{Zr} / \mathrm{Hf}=$ 22.28-59.20, $\mathrm{Nb} / \mathrm{Ta}=3.39-11.14)$.

\section{Syenites}

Red syenites are potassic $(\mathrm{K} / \mathrm{Na}=1.46-5.44)$ with high silica and high alkali $\left(\mathrm{Na}_{2} \mathrm{O}+\mathrm{K}_{2} \mathrm{O}\right)$ contents ranging from 57.49 to $71.67 \mathrm{wt} . \%$ and 6.56 to $12.40 \mathrm{wt} . \%$, respectively (Figure 4), and 1.80 to 12.78 wt.\% $\mathrm{CaO} . \mathrm{MgO}$ and $\mathrm{FeO}$ content ranges from 0.53 to 7.68 wt. $\%$ and 1.76 to 4.22 wt.\%, respectively, with $\mathrm{Mg \#}=$ 0.54-0.87 (Figure 5).

White syenites have alkali contents $\left(\mathrm{K} / \mathrm{Na}=0.19-7.51 ; \mathrm{Na}_{2} \mathrm{O}\right.$ $+\mathrm{K}_{2} \mathrm{O}=4.23-12.77$ wt.\%) (Figure 4) similar to red syenites and 52.75 to 58.47 wt.\% $\mathrm{SiO}_{2}, 6.89$ to 14.26 wt.\% $\mathrm{CaO}, 0.18$ to 2.99 wt.\% $\mathrm{MgO}(\mathrm{Mg} \#=0.40-0.72)$, and 0.69 to 7.01 wt.\% $\mathrm{FeO}$ (Figure 5). We note that one wollastonite-rich white syenite has very low alkali content $\left(2.65 \mathrm{wt} . \% \mathrm{Na}_{2} \mathrm{O}+\mathrm{K}_{2} \mathrm{O}\right)$ and very high $\mathrm{CaO}$ (33.87 wt.\%; Table 1, IC2-27, Figure 2F).

Red syenites are LREE-enriched compared to HREE on a chondrite-normalized plot (573-1,100 times; $\mathrm{La}_{\mathrm{N}} / \mathrm{Yb}_{\mathrm{N}}=$ 17.61-90.44) with negative $\mathrm{Eu}$ anomaly $\left(\mathrm{Eu} / \mathrm{Eu}^{*}=0.47-0.75\right)$ (Figure 6E). In comparison with carbonatites, they have higher

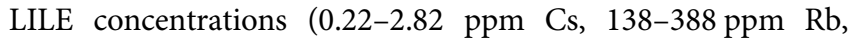
698-4,198 ppm Ba), with negative anomalies in $\mathrm{Nb}-\mathrm{Ta}$ and $\mathrm{Zr}-\mathrm{Hf}(\mathrm{Zr} / \mathrm{Hf}=13.47-29.88, \mathrm{Nb} / \mathrm{Ta}=0.16-21.51$; Figure $6 \mathrm{~F})$.

REE white syenite patterns are similar to red syenite patterns, with LREE enrichment compared to HREE (739-1862 times; La $=175-441 \mathrm{ppm} ; \mathrm{La}_{\mathrm{N}} / \mathrm{Yb}_{\mathrm{N}}=35-131.25$; Figure 6G). They have variable concentrations in Cs $(0.09-2.71 \mathrm{ppm} \mathrm{Cs})$ and $\mathrm{Rb}$ (23.5-229 ppm Rb), high Ba contents (900-13,197 ppm Ba), and negative anomalies in $\mathrm{Zr}-\mathrm{Hf}$ and $\mathrm{Nb}-\mathrm{Ta}(\mathrm{Zr} / \mathrm{Hf}=$ 16.18-54.83, $\mathrm{Nb} / \mathrm{Ta}=0.19-7.81$; Figure $6 \mathbf{H}$ ).

\section{MINERAL CHEMISTRY}

\section{Clinopyroxene}

All clinopyroxenes in carbonatites are euhedral diopside $\left(\mathrm{En}_{26-33} \mathrm{Fs}_{17-23} \mathrm{Wo}_{45-51}\right)$ or hedenbergite $\left(\mathrm{En}_{18-22} \mathrm{Fs}_{30-32} \mathrm{Wo}_{46-48}\right)$ phenocrysts (Figure 7A; Supplementary Table S3), and no dissolution/recrystallization texture or core to rim zonation has been observed. In Si-poor carbonatites, clinopyroxenes are diopsides $\left(\mathrm{En}_{28-33} \mathrm{Fs}_{17-22} \mathrm{Wo}_{48-51}\right)$ with relatively high $\mathrm{Mg} \#(\mathrm{Mg} \#=\mathrm{Mg} /[\mathrm{Mg}$ 


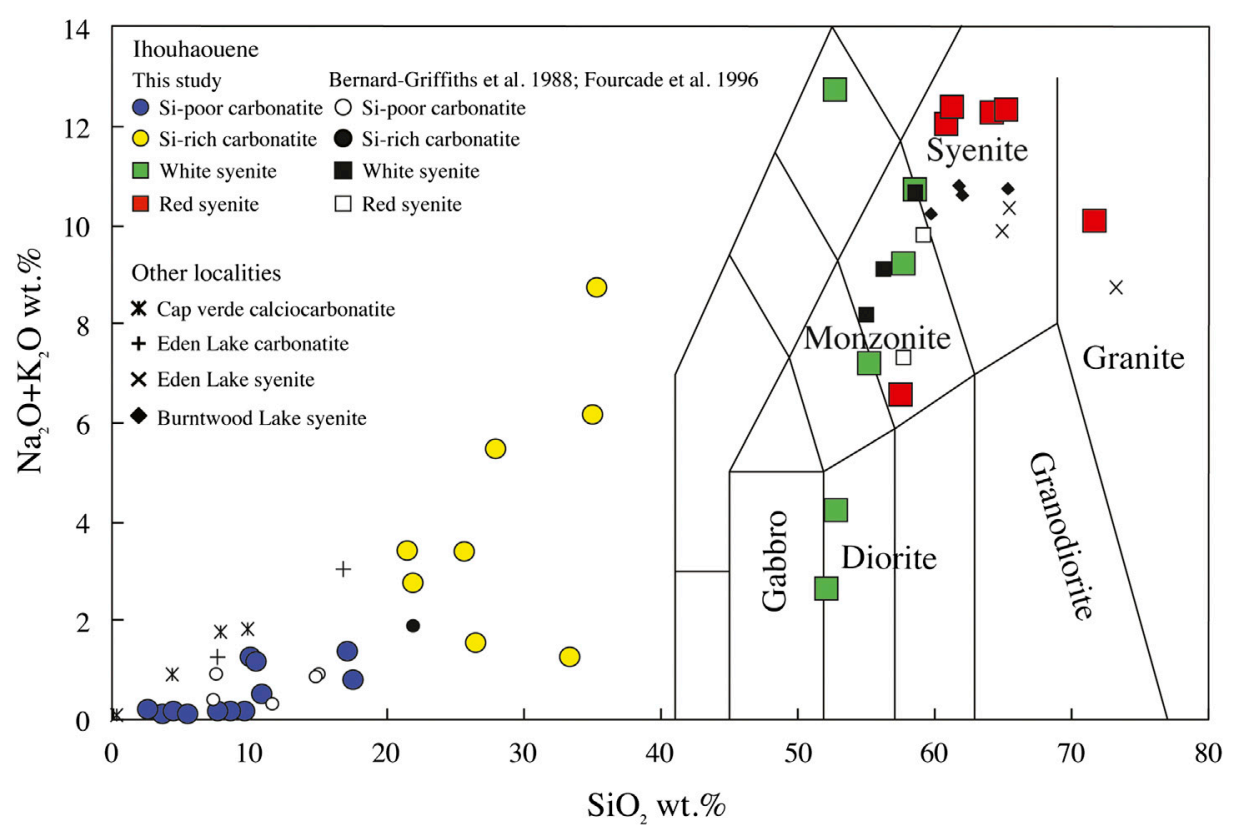

FIGURE 4 | Ihouhaouene carbonatites and associated syenites in total alkali-silica (TAS) diagram (after Le Maitre et al., 1989). Cap verde carbonatites (Weinderdorfer et al., 2016), Eden Lake carbonatites and syenites (Chakhmouradian et al., 2008), and Burntwood Lake syenites (Martins et al., 2011) are shown.
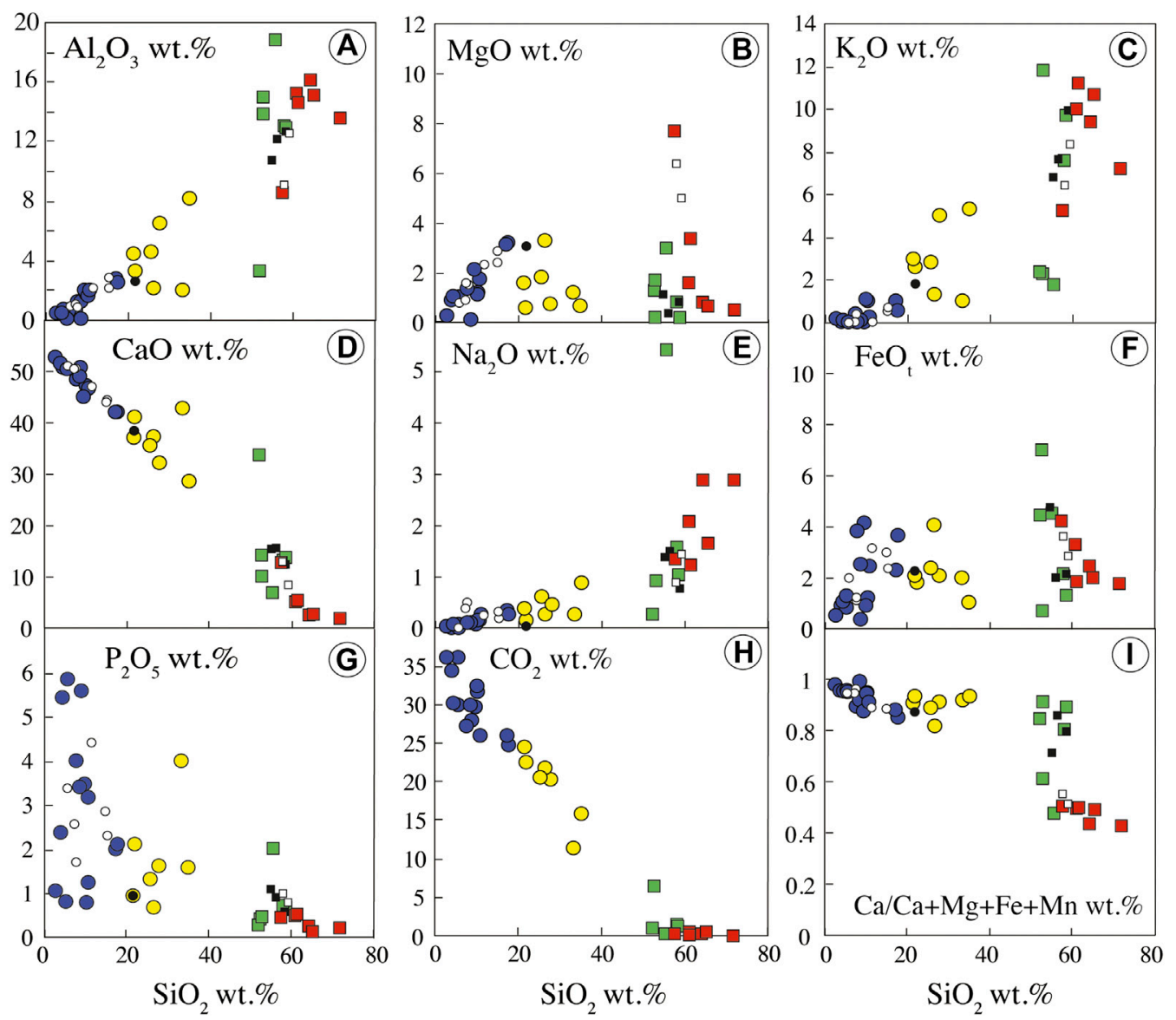

FIGURE 5 | Bulk rock major oxide compositions of the lhouhaouene carbonatites and syenites. Symbols as in Figure 4. 

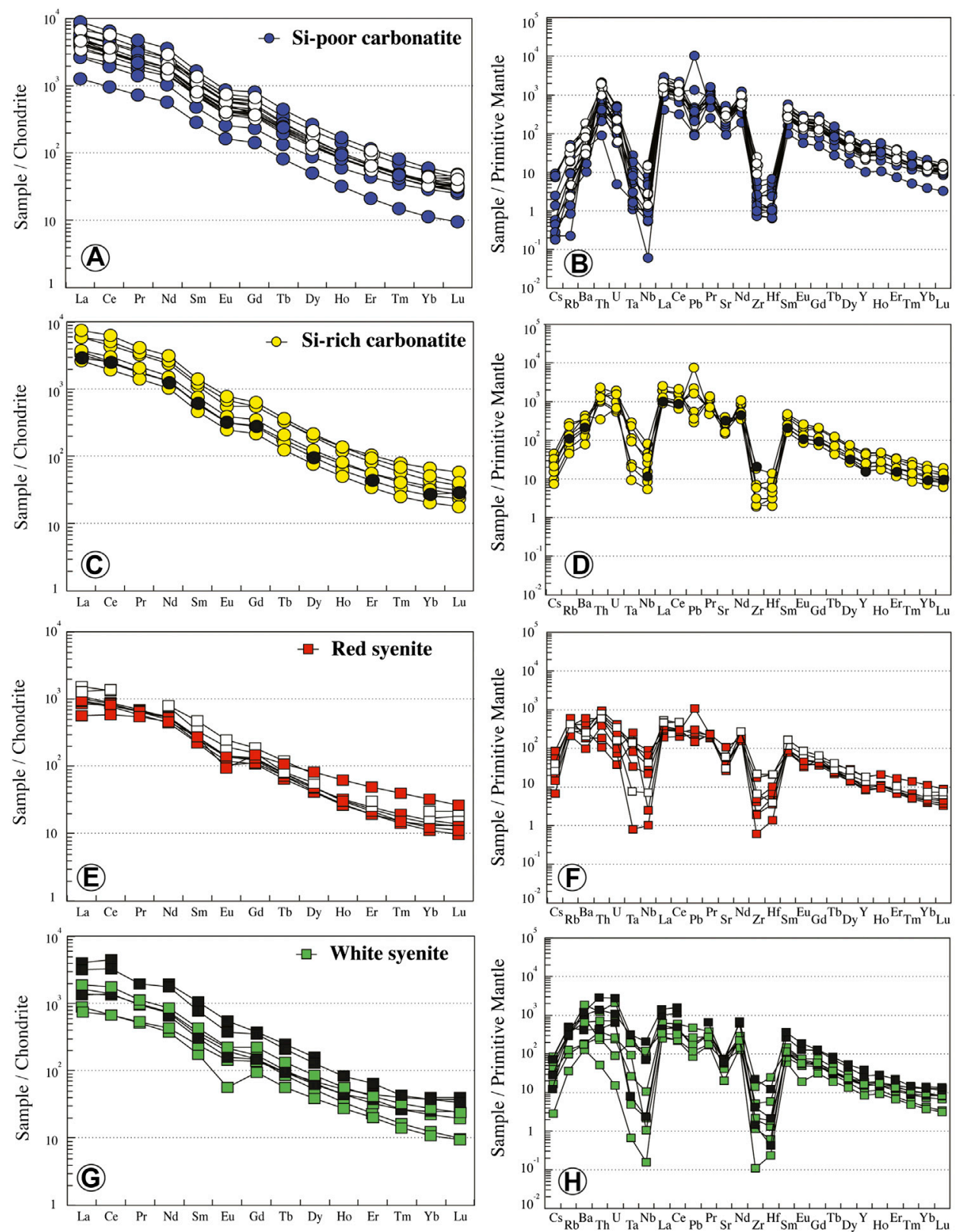

FIGURE 6 | Normalized REE and trace element contents of Ihouhaouene carbonatites and associated syenites. Primitive mantle values and chondrite values are obtained from Sun and McDonough (1989). Symbols as in Figure 4.

$\left.+\mathrm{Fe}^{2+}\right]=0.63-0.74, \mathrm{Fe}^{3+} / \mathrm{Fe}_{\mathrm{t}}<0.36$; Droop, 1987) and low $\mathrm{Cr}_{2} \mathrm{O}_{3}$, $\mathrm{Na}_{2} \mathrm{O}, \mathrm{TiO}_{2}$, and $\mathrm{Al}_{2} \mathrm{O}_{3}$ contents $\left(\mathrm{TiO}_{2}=0.01-0.32\right.$ wt. $\%, \mathrm{Al}_{2} \mathrm{O}_{3}=$ 0.37-3.67 wt.\%; Figure 7B; Supplementary Table S3). Diopsides have very low LREE concentrations $\left(\mathrm{La}_{\mathrm{N}}<1.9, \mathrm{La}_{\mathrm{N}} / \mathrm{Yb}_{\mathrm{N}}=0.13-0.76\right)$ with regular flat patterns in middle rare earth elements (MREE) $\left(\mathrm{Sm}_{\mathrm{N}} /\right.$ $\mathrm{Yb}_{\mathrm{N}}=1.03-3.78$, Figure 8A). They display positive anomalies in $\mathrm{Ba}$, $\mathrm{U}, \mathrm{Pb}$, and $\mathrm{Sr}$ and $\mathrm{Zr}-\mathrm{Hf}$ and $\mathrm{Nb}-\mathrm{Ta}$ fractionation $(\mathrm{Zr} / \mathrm{Hf}=9.26-20.05$, $\mathrm{Nb} / \mathrm{Ta}=10.33-17.37$; Figure 8B; Supplementary Table S4).

Si-rich carbonatites have both diopside $\left(\mathrm{En}_{26-32} \mathrm{Fs}_{19-23} \mathrm{Wo}_{45-50}\right)$ and hedenbergite $\left(\mathrm{En}_{18-22} \mathrm{Fs}_{30-32} \mathrm{Wo}_{46-48}\right)$ pyroxenes (Figure 7A) with $\mathrm{Mg} \#=0.55-0.77\left(\mathrm{Fe}^{3+} / \mathrm{Fe}_{\mathrm{t}}<0.29\right)$ and $0.42-0.49\left(\mathrm{Fe}^{3+} / \mathrm{Fe}_{\mathrm{t}}=\right.$
0.18-0.24), respectively (Supplementary Table S3). Diopside and hedenbergite have low $\mathrm{TiO}_{2}$ contents $\left(\mathrm{TiO}_{2}=0.02-0.33 \mathrm{wt} . \%\right.$, Figure 7B), and $\mathrm{Al}_{2} \mathrm{O}_{3}$ contents range from 0.49 to $1.42 \mathrm{wt} . \%$ and 0.22 to 1.04 wt.\%, respectively. REE patterns have a sinusoidal to asymmetric $\mathrm{U}$-shape with slightly high concentrations in LREE compared to HREE $\left(\mathrm{La}_{\mathrm{N}} / \mathrm{Yb}_{\mathrm{N}}=1.17-4.89\right.$; Figure 8C; Supplementary Table S4). Large variations in LILE concentrations are observed in Si-rich carbonatite pyroxenes with strong positive anomalies for $\mathrm{Pb}$ and $\mathrm{Sr}$ in addition to $\mathrm{Zr}$ $\mathrm{Hf}$ and $\mathrm{Nb}-\mathrm{Ta}$ fractionation $(\mathrm{Zr} / \mathrm{Hf}=20.71-33.93, \mathrm{Nb} / \mathrm{Ta}=$ 1.57-50.73; Figure 8D). 

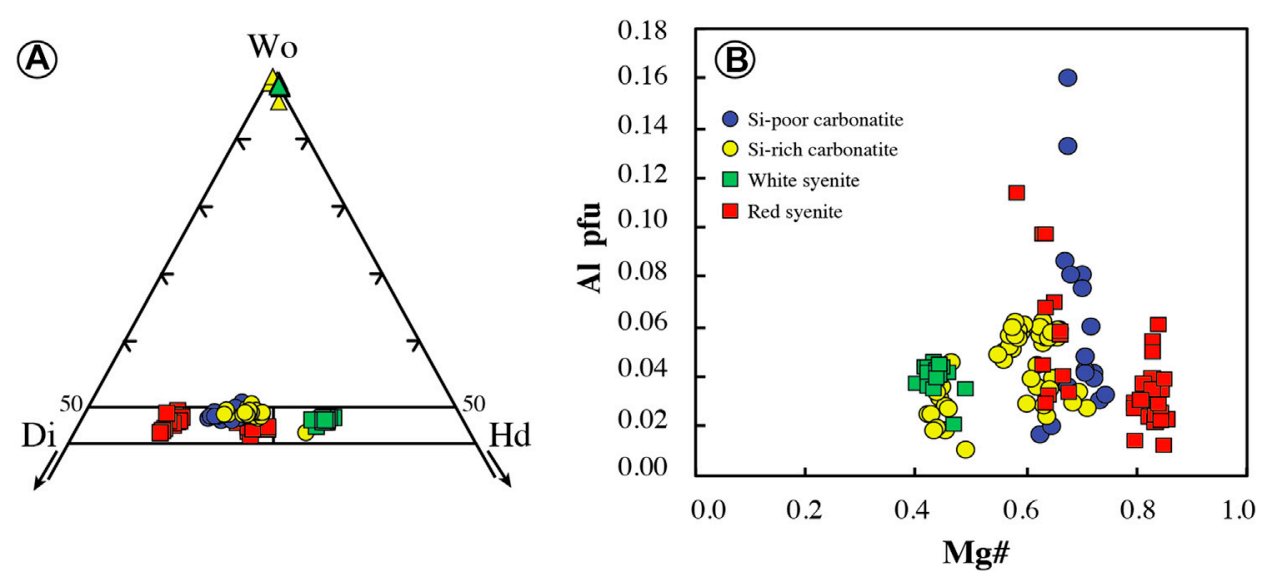

FIGURE 7 | Clinopyroxene mineral chemistry of the Ihouhaouene carbonatites and syenites in molar units. (A) Chemical variation of clinopyroxene and wollastonite (triangles) in the Enstatite (En)-Wollastonite (Wo)-Ferrossilite (Fs) ternary diagram of Morimoto, (1988). (B) Al vs. Mg\# (p.f.u.); Mg\# = Mg/(Mg + Fe tot). Symbols as in Figure 4.

In red syenites, pyroxenes are diopsides $\left(\mathrm{En}_{27-41} \mathrm{Fs}_{11-25} \mathrm{Wo}_{46-49}\right.$; Figure 7A). They have high $\mathrm{Mg} \#$ ranging from 0.63 to $0.86\left(\mathrm{Fe}^{3+} /\right.$ $\left.\mathrm{Fe}_{\mathrm{t}}<0.37\right)$ and $\mathrm{Na}_{2} \mathrm{O}=0.4-0.8$ wt.\%, $\mathrm{TiO}_{2}=0.02-0.26$ wt.\%, $\mathrm{Al}_{2} \mathrm{O}_{3}=0.28-2.21$ wt.\%, and $\mathrm{Cr}_{2} \mathrm{O}_{3}<0.05$ wt.\% (Figure $7 \mathbf{B}$; Supplementary Table S3). Chondrite-normalized patterns of diopside in red syenites display a convex LREE section $\left(\mathrm{La}_{\mathrm{N}} /\right.$ $\left.\mathrm{Sm}_{\mathrm{N}}=0.13-1.30\right)$ and a negative $\mathrm{Eu}$ anomaly $\left(\mathrm{Eu} / \mathrm{Eu}^{*}=0.11\right.$ to 0.28 ; Figure 8E; Supplementary Table S4). Relative to the primitive mantle, pyroxenes have high fractionation in $\mathrm{Nb}-\mathrm{Ta}(\mathrm{Nb} /$ $\mathrm{Ta}=1.63-28.55)$ with negative anomalies in $\mathrm{Ba}, \mathrm{Pb}, \mathrm{Sr}$, and $\mathrm{Zr}$ and enrichment in Th (Figure 8F; Supplementary Table S4). It should be noted that clinopyroxenes that crystallized around red or white syenite fragments are diopside or hedenbergite, respectively, with the same composition compared to interstitial clinopyroxene in red or white syenite.

White syenites are characterized by Fe-rich pyroxenes with hedenbergitic composition $\left(\mathrm{En}_{17-20} \mathrm{Fs}_{31-33} \mathrm{Wo}_{47-49}, \mathrm{Fe}^{3+} / \mathrm{Fe}_{\mathrm{t}}=\right.$ 0.10-0.31) (Figure 7A; Supplementary Table S3) and 0.39-0.78 wt.\% $\mathrm{Na}_{2} \mathrm{O}, \quad 0.44-1.06$ wt.\% $\quad \mathrm{Al}_{2} \mathrm{O}_{3}, \quad<0.12$ wt.\% $\mathrm{TiO}_{2}$, and $<0.03$ wt.\% $\mathrm{Cr}_{2} \mathrm{O}_{3}$ (Figure 7B; Supplementary Table S3). The REE patterns of hedenbergite in white syenites have low REE content and the same sinusoidal to asymmetric U-shaped pattern compared to Si-rich carbonatites, with high LREE and HREE concentrations compared to MREE $\left(\mathrm{La}_{\mathrm{N}} / \mathrm{Yb}_{\mathrm{N}}=0.58-3.12, \mathrm{La}_{\mathrm{N}} / \mathrm{Sm}_{\mathrm{N}}=\right.$ 0.73-2.27) (Figure 8G). They have variable trace element contents down to very low values (i.e., $\mathrm{Rb}, \mathrm{Nb}, \mathrm{Ta}, \mathrm{Pb}$, and $\mathrm{Sr}$ ) compared to pyroxenes in red syenites (Figure $\mathbf{8 H}$; Supplementary Table S4).

\section{Apatite}

Apatites in carbonatites and syenites are fluorapatites (1.8-4.5 wt.\% F, Supplementary Table S5). They have different silica, phosphate, calcium, and REE contents depending of the host rock (Figure 9; Supplementary Table S5), but despite their relatively large size (up to $5 \mathrm{~mm}$ ), apatite crystals do not have significant zonation from core to rim or dissolution/recrystallization texture.
In Si-poor carbonatites, apatite has low silica $\left(0.28-2.46\right.$ wt. $\% \mathrm{SiO}_{2}$, Figure 9) and REE contents $(\Sigma \mathrm{REE}=0.66-5.67$ wt.\% $)$ and $\mathrm{P}_{2} \mathrm{O}_{5}$ content from 34.84 to 42.05 wt.\% (Supplementary Table S5). Apatite has low LREE compared to MREE concentration with convex patterns, both of which are higher than $\operatorname{HREE}\left(\mathrm{La}_{\mathrm{N}} / \mathrm{Sm}_{\mathrm{N}}=0.35-1.70, \mathrm{La}_{\mathrm{N}} / \mathrm{Yb}_{\mathrm{N}}=\right.$ 6.31-37.65; Figure 10A). Primitive mantle-normalized patterns show high values for $\mathrm{Th}, \mathrm{U}, \mathrm{Sr}$, and $\mathrm{Pb}(1,000$ times the primitive mantle; i.e., $\mathrm{Th}=49-348 \mathrm{ppm}, \mathrm{U}=2.3-39.3 \mathrm{ppm})$ and negative $\mathrm{Nb}-\mathrm{Ta}$ and $\mathrm{Zr}$ $\mathrm{Hf}$ anomalies $(\mathrm{Nb} / \mathrm{Ta}=0.71-10.96, \mathrm{Zr} / \mathrm{Hf}=28.91-127.74$; Figure 10B; Supplementary Table S6).

In Si-rich carbonatites, apatite is silica- and REE-rich $\left(\mathrm{SiO}_{2}=2.34-6.05\right.$ wt.\%, $\quad \Sigma \mathrm{REE}=4.32-11.22 \mathrm{wt} . \%$, respectively, Figure 9). $\mathrm{P}_{2} \mathrm{O}_{5}$ content ranges from 28.67 to 37.43 wt. $\%$ and $\mathrm{CaO}$ from 47.77 to 52.40 wt.\% (Supplementary Table S5). Apatite REE patterns in Si-rich carbonatites are straight with high normalized LREE compared to HREE $\left(\mathrm{La}_{\mathrm{N}} / \mathrm{Yb}_{\mathrm{N}}=86.77-142.66\right)$ and slight negative $\mathrm{Eu}$ anomaly (Figure 10C), and primitive mantlenormalized patterns have higher $\mathrm{Th}, \mathrm{U}, \mathrm{Sr}$, and $\mathrm{Pb}$ contents ( $\mathrm{Th}=1,200-4,731 \mathrm{ppm}, \mathrm{U}=147-818 \mathrm{ppm}$ ) than apatite in Sipoor carbonatite and low negative $\mathrm{Nb}-\mathrm{Ta}$ and $\mathrm{Zr}-\mathrm{Hf}$ anomalies $(\mathrm{Nb} / \mathrm{Ta}=33.66-177.42, \mathrm{Zr} / \mathrm{Hf}=102.35-241.32$; Figure 10D; Supplementary Table S6).

Apatite in red syenites has low silica contents $\left(\mathrm{SiO}_{2}=\right.$ 0.23-1.67 wt.\%) with 40.03-46.89 wt.\% $\mathrm{P}_{2} \mathrm{O}_{5}$ and 53.07-56.72 wt.\% $\mathrm{CaO}$ with total REE from 0.50 to 3.82 wt.\% (Supplementary Table S5; Figure 9). REE patterns are MREE-enriched compared to LREE $\left(\mathrm{La}_{\mathrm{N}} / \mathrm{Sm}_{\mathrm{N}}=0.14-0.78, \mathrm{La}_{\mathrm{N}} / \mathrm{Yb}_{\mathrm{N}}=1.59-12.67\right)$ with a convex pattern and conspicuous negative anomaly in $\mathrm{Eu}\left(\mathrm{Eu} / \mathrm{Eu}^{\star}=0.07\right.$ to 0.24 ; Figure 10E). Negative anomaly is observed for $\mathrm{Nb}-\mathrm{Ta}$ and $\mathrm{Zr}-\mathrm{Hf}$ fractionation in primitive mantle-normalized patterns $(\mathrm{Nb} / \mathrm{Ta}=$ 5.14-83.45, $\mathrm{Zr} / \mathrm{Hf}=5.77-127.78$; except for one sample $\mathrm{Zr} / \mathrm{Hf}=$ 446.66; Supplementary Table S6; Figure 10F).

White syenites have silica-rich apatites with 1.73-3.49 wt.\% $\mathrm{SiO}_{2}, 33.39-43.03$ wt.\% $\mathrm{P}_{2} \mathrm{O}_{5}$, and 50.78-54.10 wt.\% $\mathrm{CaO}$ and intermediate REE content (2.2-8.0 wt.\% total REE) (Supplementary Table S5; Figure 9). REE patterns of apatite in white syenites display the same regular shape compared to Si-rich 

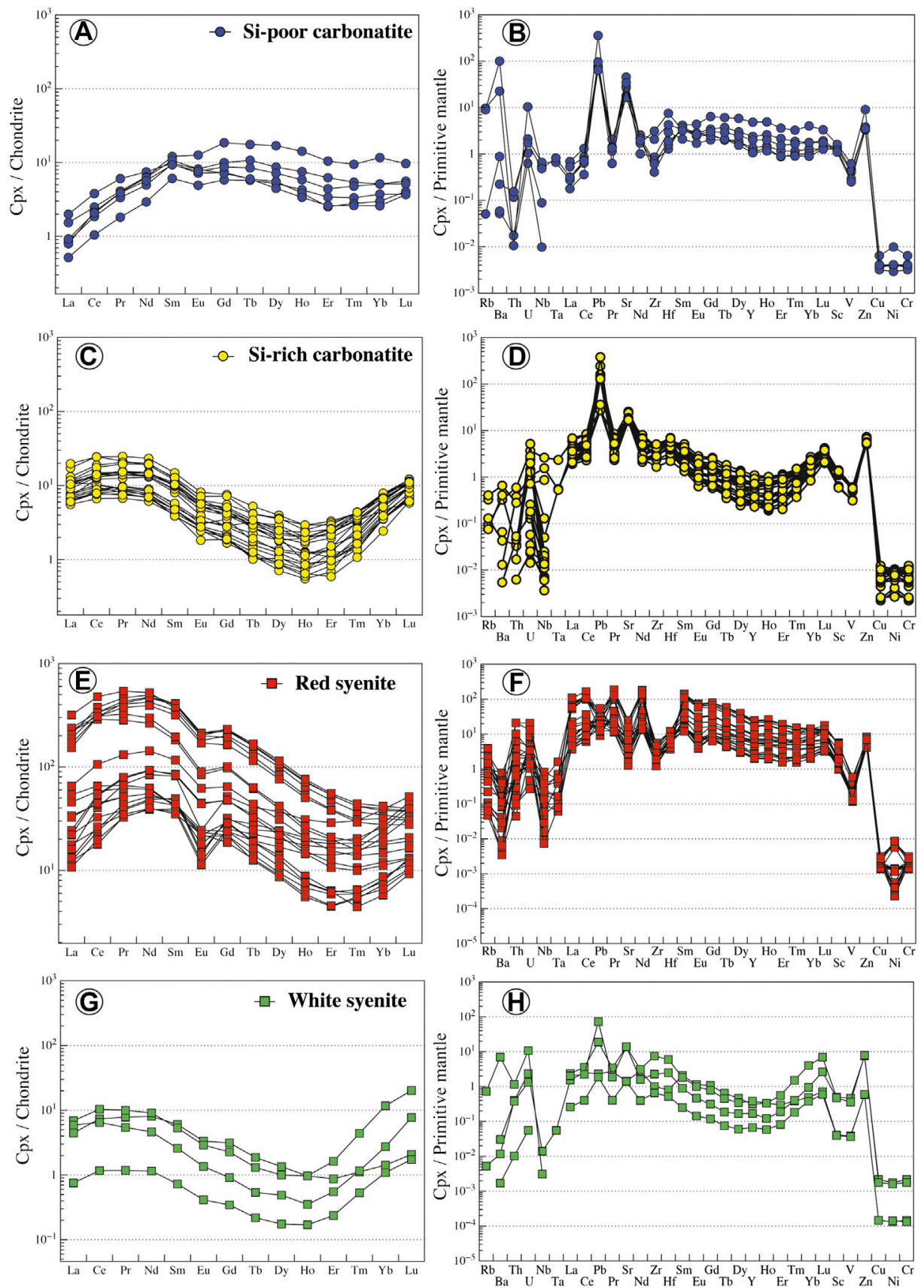

FIGURE 8 | Normalized REE and trace element contents of clinopyroxene from Ihouhaouene carbonatites and associated silicate rocks. Chondrite values and primitive mantle values are obtained from Sun and McDonough (1989).

apatite patterns with high concentrations of normalized LREE compared to HREE $\left(\mathrm{La}_{\mathrm{N}} / \mathrm{Yb}_{\mathrm{N}}=76.39-168.24\right.$; Figure 10G). They have high content in $\mathrm{Th}(\mathrm{Th}=372-4,984 \mathrm{ppm})$ and negative $\mathrm{Zr}-\mathrm{Hf}$ and $\mathrm{Nb}-\mathrm{Ta}$ anomalies $(\mathrm{Nb} / \mathrm{Ta}=54.09-98.46, \mathrm{Zr} / \mathrm{Hf}=$ 97.66-232.34; Supplementary Table S6; Figure 10H).

\section{Wollastonite}

Wollastonite is present in Si-rich carbonatites and white syenites and has a homogeneous composition (En0-
0.05Fs1-2W097-99, Figure 8B; Supplementary Table S7). It has flat regular patterns and small negative Eu anomaly in carbonatite and syenite $\left(10-50^{\star}\right.$ Chondrite, LaN/YbN = 0.11-0.56). However, altered wollastonite in carbonatite displays a concave REE pattern with high LREE content $(\mathrm{LaN} / \mathrm{YbN}=9.16-10.42 ;$ Figure 11A). Wollastonite in white syenites has a higher negative anomaly in $\mathrm{Nb}-\mathrm{Ta}$ than in carbonatites $(\mathrm{Nb} / \mathrm{Ta}=0.76-1.53$ and $0.21-18.94$, respectively, Supplementary Table S8). 


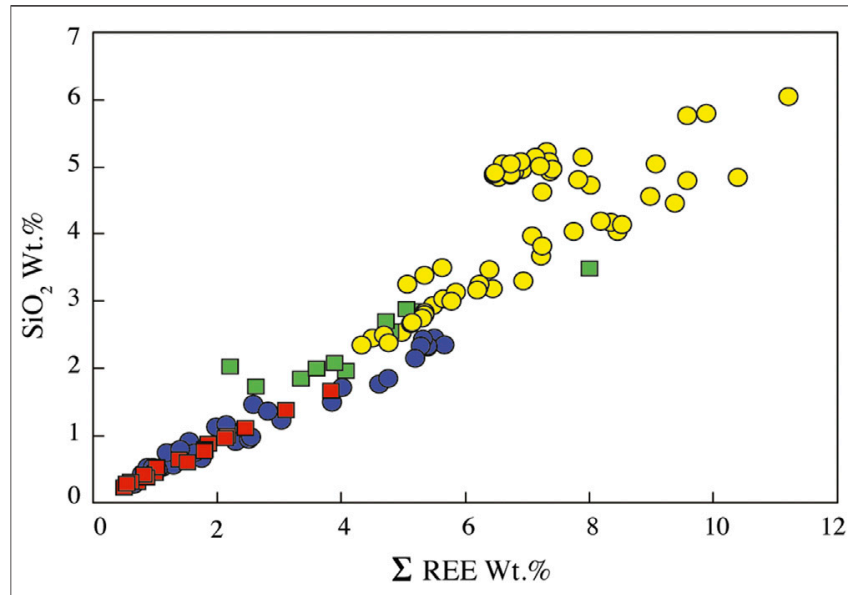

FIGURE 9 | $\mathrm{SiO}_{2}$ vs. REE $\mathrm{Rx}_{\text {wt. }}$ w of apatite from the lhouhaouene carbonatites and syenites. Symbols as in Figure 4.

\section{Carbonates}

Carbonate in the all carbonatites of Ihouhaouene is calcite $(\mathrm{CaO}=$ 56-59 wt.\%, $\mathrm{MgO}<0.16$ wt.\%, $\mathrm{FeO}<0.15, \mathrm{MnO}=0.01-0.2$ wt.\%, $\mathrm{Sr}=3,653-6,938 \mathrm{ppm}$, Supplementary Tables S7, and S8). REE patterns of calcite in Si-poor carbonatites have low LREE concentrations $\left(\mathrm{La}_{\mathrm{N}} / \mathrm{Yb}_{\mathrm{N}}=0.13-0.16\right)$, whereas calcites in Sirich carbonatites have high LREE contents compared to HREE $\left(\mathrm{La}_{\mathrm{N}} / \mathrm{Yb}_{\mathrm{N}}=14.06-17.16\right.$, Figure 11B; Supplementary Table S8).

\section{Alkali Feldspar}

In red and white syenites, the alkali feldspars are potassic with $\mathrm{Or}_{57-96} \mathrm{Ab}_{3-41} \mathrm{An}_{1-2}$ contents and have perthitic exsolutions with $\mathrm{Or}_{2-14} \mathrm{Ab}_{66-98} \mathrm{An}_{0-20}$. In Si-rich carbonatites, alkali feldspars have composition close to feldspar in syenite with high orthoclase content of $\mathrm{Or}_{95-98}$ and $\mathrm{BaO}$ content (Supplementary Tables S7, and S8).

\section{Accessory Minerals}

Accessory minerals ( $<1$ vol.\%) are present as interstitial minerals, corona, or small inclusions in some samples (Supplementary Table S1; Section 3) with texture of crystallization at subsolidus conditions. For clarity, we present only a brief overview of the texture and composition of accessory minerals as they represent very small volume of the rocks and do not significantly change the composition of magmatic minerals and bulk rocks. It is important to note that inclusion-free and inclusion-bearing apatites have the same composition and associated clinopyroxenes are unzoned with homogenous composition in each rock facies.

\section{Britholite}

Britholite is a REE mineral isostructural with apatite with general formula $(\mathrm{Na}, \mathrm{Ca}, \mathrm{REE})_{10}(\mathrm{Si}, \mathrm{P})_{6} \mathrm{O}_{24}(\mathrm{OH}, \mathrm{F})_{2}$ and $\mathrm{Si}>\mathrm{P}$ (Oberti et al., 2001). They are present in one Si-rich carbonatites (plus one Si-poor sample) and white syenites as fine lamellar exsolutions ( $<10 \mathrm{um})$ or as irregular-shaped grains (10-200 um) in apatite (Anenburg et al., 2018; Lorenz et al., 2019). The apatite exsolutions correspond to a britholite substitution of $\mathrm{Ca}^{2+}$ and $\mathrm{P}^{5+}$ for $\mathrm{REE}^{3+}$ and $\mathrm{Si}^{4+}$, and the very low $\mathrm{Na}$ content indicates no belovite substitution. Britholites have 8-16 wt.\% $\mathrm{La}_{2} \mathrm{O}_{3}, 21-43$ wt.\% $\mathrm{Ce}_{2} \mathrm{O}_{3}$, and 7-12 wt.\% $\mathrm{Nd}_{2} \mathrm{O}_{3}$ and contain up to 60 wt. $\%$ REE, dominated by LREE $\left(\mathrm{La}_{\mathrm{N}} / \mathrm{Yb}_{\mathrm{N}}=\right.$ 157-318; Supplementary Tables S7, and S8).

\section{Monazite}

Monazite is likely also exsolution (Anenburg et al., 2018; Lorenz et al., 2019); it is present as inclusions in apatite and around apatite crystals in Si-poor carbonatites and red syenites. It has more than $60 \%$ of REE with high $\mathrm{La}$ and Ce concentrations ( $\mathrm{La}=16.53-23.34$ wt.\%, Ce = 32.52-36.01 wt.\%; Supplementary Table S7).

\section{Allanite}

Present in all rock facies, allanite is a calcic and rareearth-bearing epidote-group mineral, with $\mathrm{SiO}_{2}$ (31.82-33.01 wt.\%), $\mathrm{CaO} \quad\left(12.73-14.93\right.$ wt.\%), $\quad \mathrm{Al}_{2} \mathrm{O}_{3}$ (11.39-15.98 wt.\%), $\mathrm{FeO}$ (10.4-16.94 wt.\%), and $\Sigma \mathrm{REE}$ (18.76-22.44 wt.\%; Supplementary Table S7). Allanites have higher LREE than HREE contents $\left(\mathrm{La}_{\mathrm{N}} / \mathrm{Yb}_{\mathrm{N}}=2,179-10,913\right.$, Supplementary Table S8) and a positive anomaly in Th (Th = $892.61 \mathrm{ppm}$ ) and $\mathrm{Nb}-\mathrm{Ta}$ and $\mathrm{Zr}$-Hf fractionation.

\section{Garnet}

Garnet has intermediate composition between grossular (63-72\%) and andradite (25-33\%; Supplementary Table S7) with low $\mathrm{TiO}_{2}$ content (0.01-0.78 wt.\%) and high $\mathrm{FeO}$ content (15.37-26.14 wt.\%). Titanian andradite-rich garnet with $\mathrm{Fe}^{3+}>\mathrm{Ti}$ is referred to as melanite (Deer et al., 1982).

\section{Magnetite and Titanite}

Oxide in carbonatites and syenites is magnetite $(\mathrm{FeO}=$ 91.56-93.98 wt.\%; Supplementary Table S7). Titanite is present as an accessory mineral in half of carbonatite and syenite samples. It has 27.64 to $29.83 \mathrm{wt} . \% \mathrm{SiO}_{2}, 31.06$ to 35.03 wt. $\% \mathrm{TiO}_{2}, 25.13$ to 17.36 wt.\% $\mathrm{CaO}$, and 1.37 to 3.25 wt.\% FeO (Supplementary Table S7). Titanite has elevated $\mathrm{Nb}$ and $\mathrm{Ta}$ contents $(\mathrm{Nb}=2,924 \mathrm{ppm} ; \mathrm{Ta}=803 \mathrm{ppm}$; Supplementary Table S8).

\section{DISCUSSION}

\section{The Ihouhaouene Complex: Case Study for the Association of Carbonatites With Silica-Saturated Syenites}

When associated with alkaline silicate rocks, carbonatites worldwide are mostly associated with silica-undersaturated rocks (e.g., Brava Island, Cape Verde-Weidendorfer et al., 2016; Kovdor, Russia-Verhulst et al., 2000); only thirty-two occurrences are distinguished by their association with silicasaturated rocks (i.e., 8\% of carbonatites worldwide-Woolley and Kjarsgaard, 2008). Among those, Mountain Pass, California (Olson et al., 1954), Oka, Canada (Eby, 1975; Chen and Simonetti, 2013), and Eden Lake, Canada (Chakhmouradian et al., 2008) show the association of calciocarbonatites with silica-saturated syenites and are furthermore comparable to Ihouhaouene in 

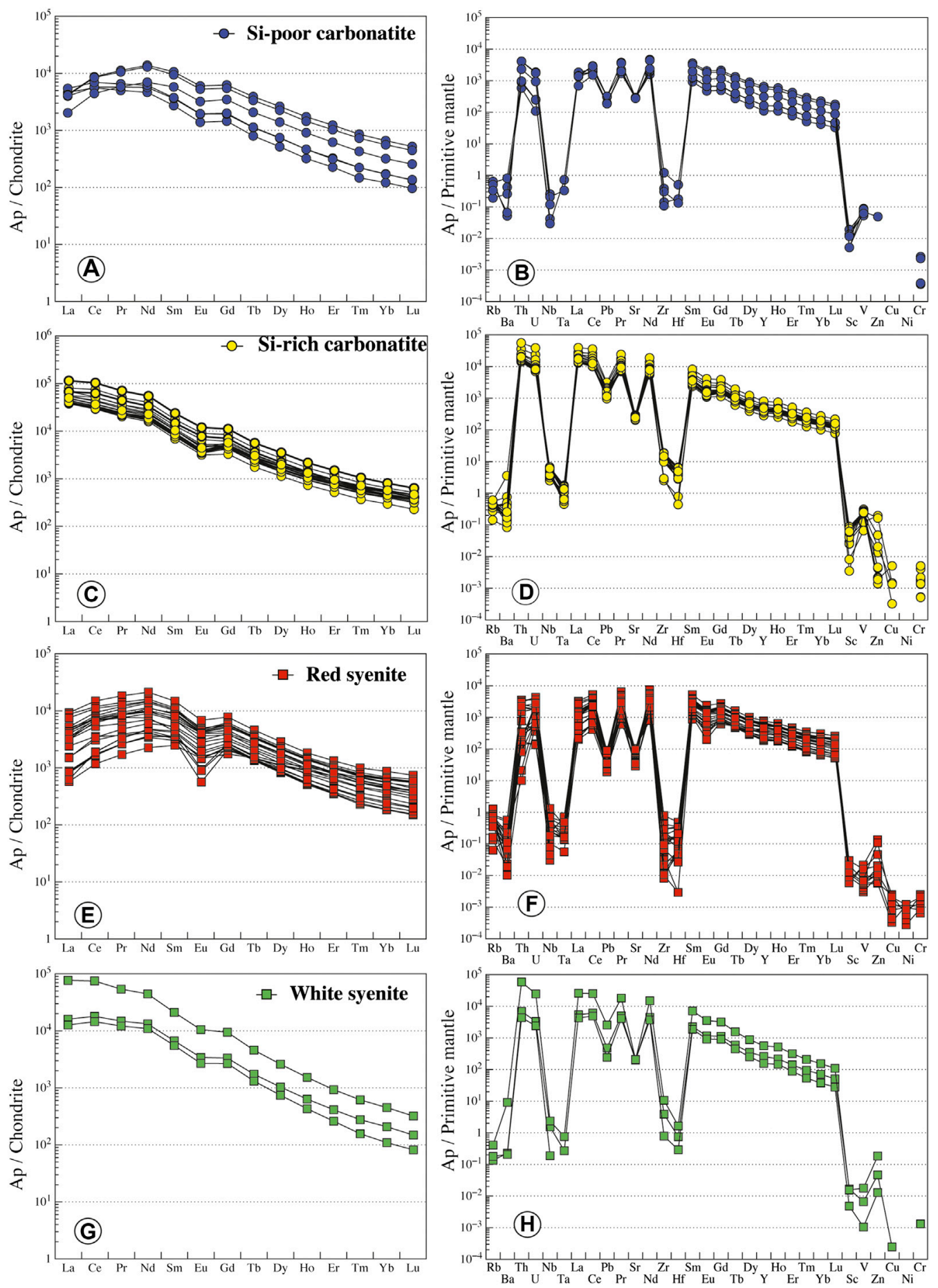

FIGURE 10 | Normalized REE and trace element contents of apatite from lhouhaouene carbonatites and associated silicate rocks. Chondrite values and primitive mantle values are obtained from Sun and McDonough (1989).

terms of mineral assemblages (diopside-hedenbergite, apatite) and major and trace element compositions (Figure 4).

In most cases, a cogenetic origin has been assumed for carbonatite and silicate rocks, whether saturated or undersaturated. However, the original magmatic features were often obscured by late hydrothermal/metamorphic imprints, precluding full understanding of petrogenetic relationships between rock facies (e.g., Jones and Wyllie, 1985; Ramasamy, 1986; Smith et al., 2000; Chakhmouradian et al., 2008; Saha et al., 2011). In the Ihouhaouene complex, the 

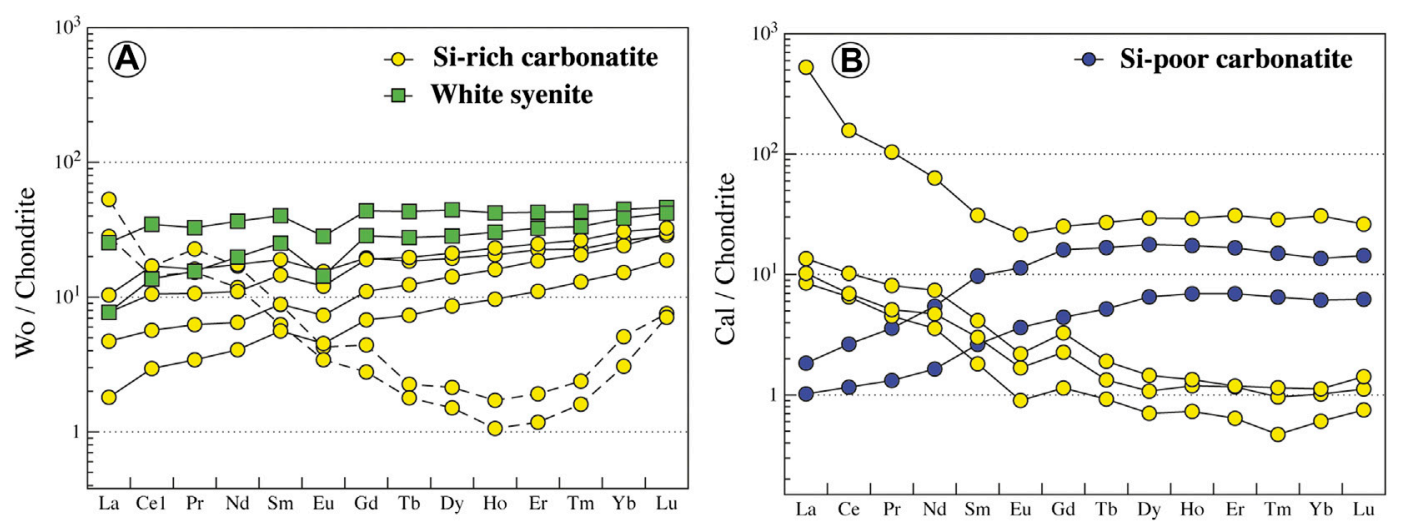

FIGURE 11 | Normalized REE content of wollastonite and calcite from Ihouhaouene carbonatites and associated silicate rocks. Chondrite values are obtained from Taylor and McLennan (1985). Altered wollastonite is shown in dashed lines.

coexistence of silica-saturated syenites with carbonatites characterized by a wide range of silica content (3-35 wt.\% $\mathrm{SiO}_{2}$ ) and the lack of significant postmagmatic imprint makes it possible to address the relationships between these rock types and shed light on the origin of the syenite-carbonatite association. We discuss in the following the salient features of the Ihouhaouene complex that should be considered in a petrogenetic model for the rock suite. This includes the geological framework of the intrusions, petrographic observations, and trace element systematics of rock-forming minerals and equilibrium melts.

\section{Geological and Isotopic Evidence for a Single Igneous Event and a Common Magma Source for Carbonatites and Syenites}

The Ihouhaouene carbonatites and syenites are closely associated in the field, where they form elongated bodies emplaced along major shear zones (Ouzegane et al., 1988; Ouzegane et al., 2003). In several complexes worldwide, the same pathway has been used by more than one magmatic event (e.g., Eifel, Vogelsberg, Kaiserstuhl-Jung et al., 2005; Walter et al., 2018; Giebel et al., 2019). In Ihouhaouene, however, carbonatites and syenites are intermingled at various scales and display similar pegmatitic/brecciated textures (Figure 2) and mineral compositions, suggesting that they were emplaced during a single event. Cogenetism of carbonatites and syenites is also supported by the parallelism between pervasive igneous layering in the complex and ductile flow foliation in the host granulite, indicating that the whole alkaline complex behaved coherently during late crystallization stages (Ouzegane et al., 1988; Ouzegane et al., 2003).

Finally, carbonatites and syenites have similar $\mathrm{Nd}, \mathrm{Sr}$, and $\mathrm{C}$ isotopic signatures consistent with a mantle origin $\left(\varepsilon \mathrm{Nd}_{(\mathrm{T})}=-6.3\right.$ to $-9.9 \%{ }_{0}{ }^{87} \mathrm{Sr} /{ }^{86} \mathrm{Sr}_{(\mathrm{T})}=0.7093$ to $0.7104, \delta^{13} \mathrm{C}=-3.5-9.7 \% 0-$ Bernard-Griffiths et al., 1988; Ouzegane et al., 1988; Fourcade et al., 1996). These values also indicate that the Ihouhaouene parental magmas were not significantly affected by granulite contamination, even though carbonate melt intrusion into a tectonically active setting can potentially result in the assimilation of fragmented wall-rock materials (Chakhmouradian et al., 2008; Giebel et al., 2019).

\section{Similar Mineral Assemblages as Evidence for Close Relationships Between Carbonatites and Syenites Throughout Parental Melt Differentiation}

One of the most conspicuous features of the Ihouhaouene complex is the presence of texturally and compositionally identical mineral assemblages (chiefly pyroxenes and apatite) occurring in both carbonatites and syenites. This is particularly true for the silica-rich carbonatites and the white syenites (Figures 7, 9) that differ from each other only by the presence of carbonates in the carbonatite while the white syenite contains alkali feldspar instead. Other mineralogical phases (clinopyroxene, wollastonite, and apatite) are almost indistinguishable in both rock types. This observation strongly supports a close cogenetic relationship between the two rock types. However, the presence of silicate minerals in carbonatites is rather unusual and must be addressed as it is most likely a key feature for deciphering the relationship between carbonatites and syenites.

Silicate minerals in carbonatites were sometimes ascribed to subsolidus recrystallization or interpreted as xenocrysts entrained by the magma (Barker, 2001; Giebel et al., 2019). Secondary-likely subsolidus-reactions are observed in the Ihouhaouene complex. Their imprint is however restricted to the development of narrow garnet coronae after K-felspar or pyroxene, crystallization of accessory minerals such as allanite, or exsolution of britholite in apatite. By no means can these processes explain the common occurrence of subhedral clinopyroxenes in the Ihouhaouene carbonatites. Since they show no evidence of resorption or zonation nor any reaction rims in contact with calcite or apatite, the clinopyroxenes are also unlikely to represent xenocrysts. Rather, they represent primary, igneous minerals crystallized during the magmatic evolution and solidification of the Ihouhaouene complex. 


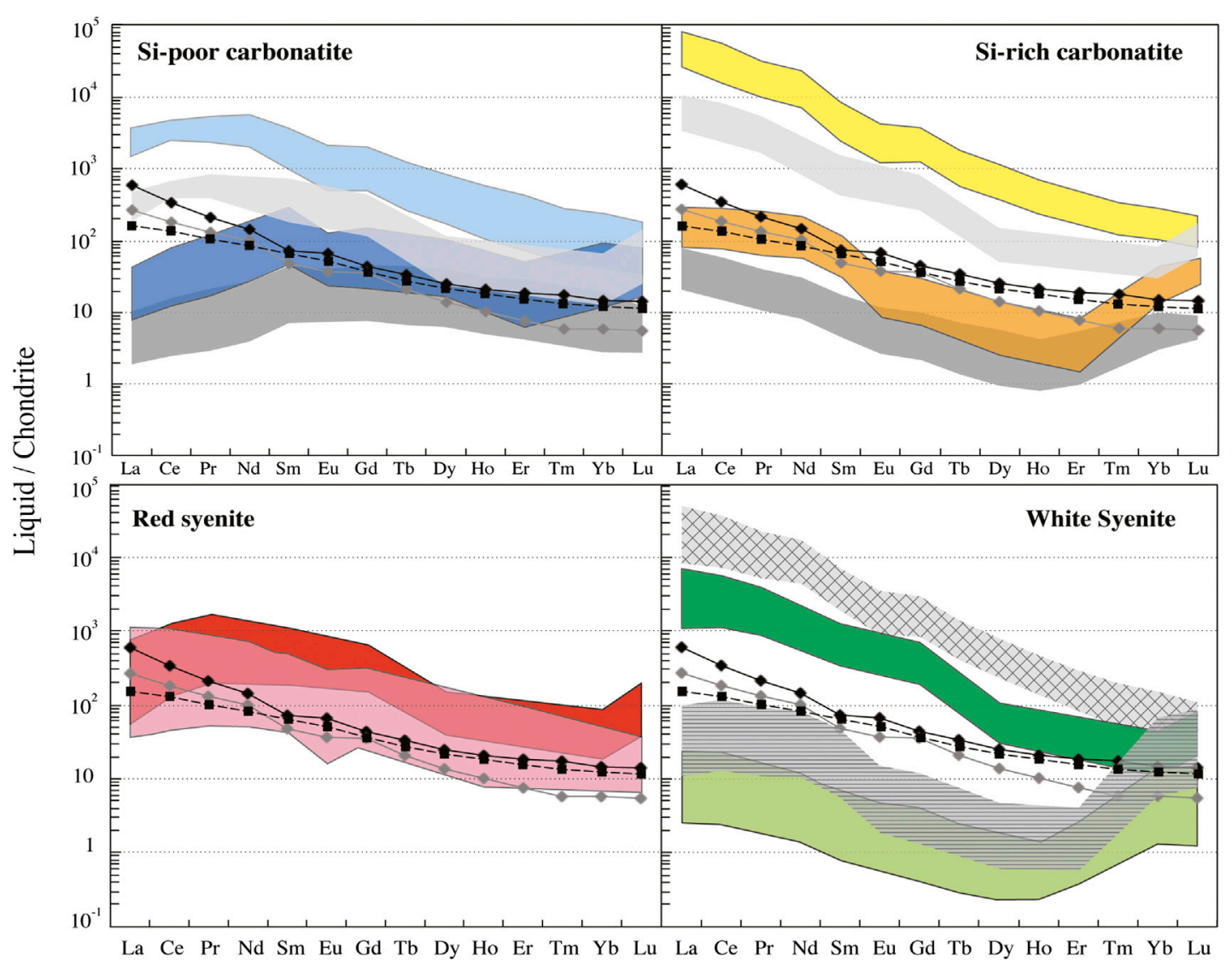

Kd Mineral/Trachyte

Ap Cpx
$\square \quad$ Red syenite
$\square$ White syenite
Carbonatite

Kd Mineral/Carbonatite

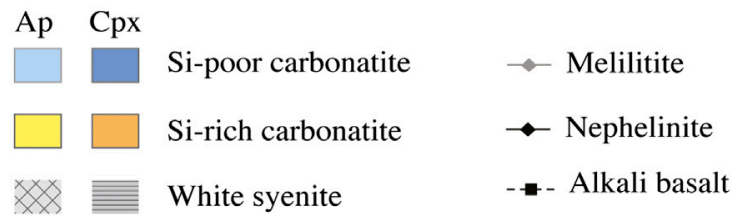

FIGURE 12 | Chondrite normalized REE contents of recalculated apatite and clinopyroxene equilibrium liquids from Ihouhaouene carbonatites and associated syenites compared to nephelinite, melilitite, and alkali basalt REE compositions. Chondrite and alkali basalt values are obtained from Sun and McDonough (1989). Hanang nephelinite and Labait melilitite are obtained from Baudouin et al. (2016) and Baudouin and Parat (2020).

A primary, igneous origin of minerals in the Ihouhaouene carbonatites is further attested by their trace element composition. The chondritic-to-near-chondritic $\mathrm{Y} / \mathrm{Ho}$ ratio in clinopyroxene (23-30), wollastonite (27-34), apatite (27-32), and calcite (28-40) in both carbonatites and syenites strongly suggests a magmatic origin at high temperature and crystallization over a temperature range evident by the increasing $\mathrm{Y} / \mathrm{Ho}$ ratios in calcite (e.g., Chakhmouradian et al., 2017; Anenburg et al., 2020). However, while the Si-rich carbonatites contain hedenbergitediopside $(\mathrm{Mg} \#=0.42-0.77-$ Figure 7$)$ associated with Si-rich apatite and wollastonite (Figures 9, 11), the Si-poor carbonatites contain diopsides $(\mathrm{Mg} \#=0.63-0.72)$ associated with $\mathrm{Si}$-poor apatite. This feature corroborates the sensitivity of silicate mineral stability and composition in carbonatites to silica activity that is usually low (Barker, 2001; Massuyeau et al., 2015), whereas the crystallization of wollastonite and hedenbergite is expected in a highly silica and calcic carbonatite with slightly low magnesium activity (Anenburg and Mavrogenes, 2018). The crystallization of hedenbergite over diopside in all rock facies is related to different $\mathrm{Mg}$ and $\mathrm{Fe}^{2+}$ activities. It should be noted that all clinopyroxene contain the same high $\mathrm{Fe}^{3+}$ content indicating similar oxidation state during crystallization.

Silica activity in carbonatites can increase as a result of assimilation of cogenetic igneous silicate rocks or contamination by country rocks at high temperature, recently described as "antiskarn metasomatism" (Chakhmouradian et al., 2008; Anenburg and Mavrogenes, 2018; Giebel et al., 2019; Anenburg et al., 2020). Due to the close intermingling of 


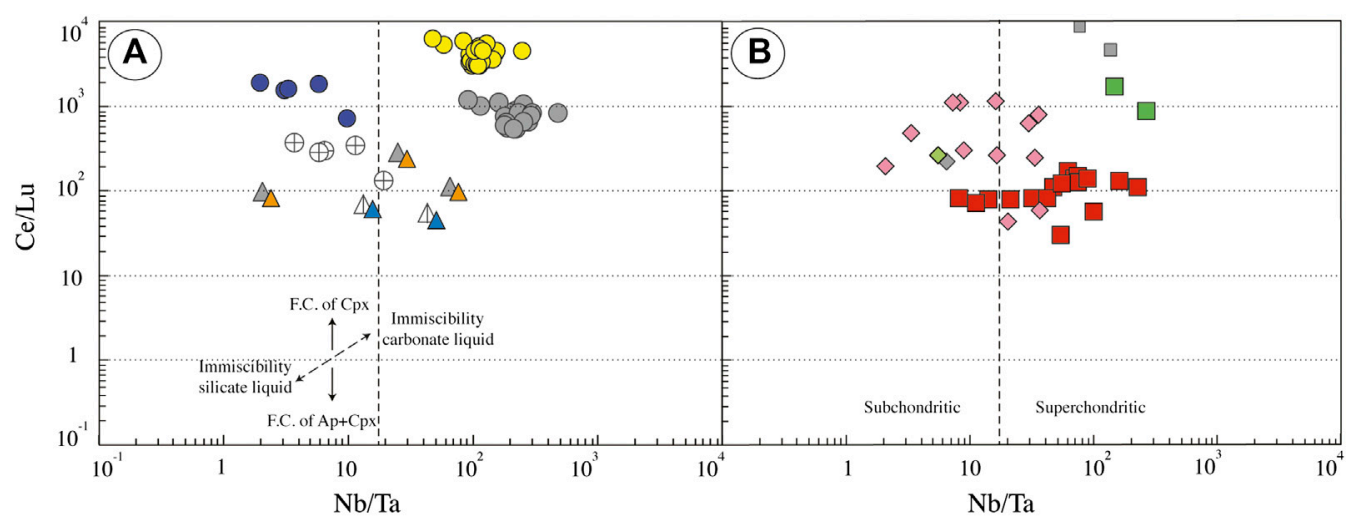

$\begin{array}{ll}\text { Kd Mineral/Trachyte } & \\ \text { Ap } & \mathrm{Cpx} \\ \square \text { White syenite } & \diamond \text { White syenite } \\ \square \text { Red syenite } & \diamond \text { Red syenite } \\ \oplus \text { Si-poor carbonatite } & \triangle \text { Si-poor carbonatite } \\ \bigcirc \text { Si-rich carbonatite } & \triangle \text { Si-rich carbonatite }\end{array}$

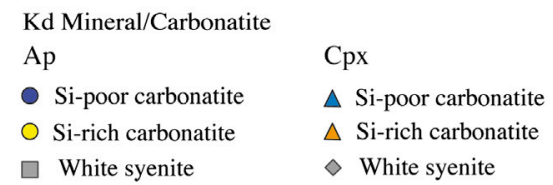

FIGURE 13 | Calculated equilibrium liquids from apatite (ap) and clinopyroxene (cpx) composition. (A) Ce/Lu vs. Nb/Ta of Ihouhaouene carbonatites. (B) Ce/Lu vs. $\mathrm{Nb} / \mathrm{Ta}$ of associated syenites. FC: fractional crystallization. For Si-poor and Si-rich carbonatite, we chose partition coefficient between carbonatite and apatite from Hammouda et al. (2010) (experiments performed with green apatite from lhouahouene) and carbonatite and clinopyroxene from Klemme et al. (1995) and Blundy and Dalton (2000). For syenites, we selected partition coefficients between silica-rich melt and apatite from Mahood and Stimac (1990) and Prowatke and Klemme (2006) and partition coefficients between silica-rich melt and clinopyroxene from Larsen (1979) and Mahood and Stimac (1990).

syenites and carbonatites in the complex and the existence of brecciated textures, the Ihouhaouene carbonatites often contain syenite fragments. These fragments may be surrounded by aureoles of clinopyroxene with the same composition as euhedral pyroxene crystals, but no further evidence for syenite assimilation was observed. In particular, the syenite fragments display no trace of zoning as would be expected for reaction with carbonatite. Although contamination by the syenite cannot be definitely excluded, the relatively high silica activity required to crystallize clinopyroxene in the Ihouhaouene carbonatites was most likely achieved by differentiation and unmixing from a carbonate-rich primary silicate melt, as previously suggested by Dawson (1998) and Baudouin et al. (2016).

\section{Trace Elements Reveal Some Complexity in the Petrogenetic Evolution of the Igneous Suite}

Trace element distribution in the Ihouhaouene complex shows several features that cannot be straightforwardly explained by a simple scheme of differentiation and unmixing from a single parental melt. These features notably include

(1) The extremely wide range of $\mathrm{Nb} / \mathrm{Ta}$ ratios in minerals, from subchondritic-to-superchondritic values (0.7-177 in apatite and clinopyroxene; $\mathrm{Nb} / \mathrm{Ta}_{\text {chondrite }}=18$-Sun and $\mathrm{McD}$ - Sonough, 1989). Both partial melting of heterogeneously carbonated mantle and separation of immiscible carbonate melts from silicate melts have been advocated to explain substantial NbTa fractionation (Koster van Groos and Wyllie, 1968; Wyllie and
Huang, 1975; Freestone and Hamilton, 1980; Kjarsgaard and Peterson, 1991; Dalton and Wood, 1993; Kjarsgaard et al., 1995; Mitchell, 2009; Brooker and Kjarsgaard, 2011; Martin et al., 2013; Novella et al., 2014). These processes would notably account for the elevated, superchondritic $\mathrm{Nb} / \mathrm{Ta}$ values frequently observed in carbonatites. In the Ihouhaouene complex, however, both carbonatites and syenites show mostly subchondritic $\mathrm{Nb} / \mathrm{Ta}$ values, with paradoxically somewhat lower values in carbonatites (0.5-17.3- Table 1) than in syenites (4.2-21.5). However, considering the unusual mineralogy and major element chemistry of many carbonatites, as well as their coarse grain size in several instances, the Ihouhaouene wholerock compositions are probably largely governed by the accumulation of "cumulus" phases, such as apatite and clinopyroxene, and accessory titanite. Therefore, these compositions are unlikely to represent true parental melt compositions. As regards to $\mathrm{Nb}-\mathrm{Ta}$, the variability of parental melts is probably more adequately reflected by the wide range of $\mathrm{Nb}$ /Ta values registered in minerals. Most samples, including the syenites, contain high $\mathrm{Nb} / \mathrm{Ta}$ minerals (Figures 8, 10) that were likely equilibrated with carbonate melts.

(2) The paradoxically low LREE content minerals of the Si-poor carbonatites compared with other rock types (Figures 8, 10). Carbonate-rich melts produced by low melting degree of a carbonated mantle source or by unmixing of carbonate melt from primary silicate melt are expected to be LREE-enriched (Hamilton et al., 1989; Jones et al., 1995; Martin et al., 2013), whereas recent experimental studies point out the role of calcium and silica activities in REE exchange reactions and mineral compositions. In clinopyroxene, the incorporation of 
LREE occurs primarily in the Ca site, $\mathrm{Ca}^{2+}+\mathrm{Si}^{4+}=\mathrm{La}^{3+}+\mathrm{Al}^{3+}$ (Anenburg et al., 2020), whereas HREE can enter in the $\mathrm{Mg}$ site, $\mathrm{Mg}^{2+}+\mathrm{Si}^{4+}=\mathrm{Lu}^{3+}+\mathrm{Al}^{3+}$ (Olin and Wolff, 2010; Dygert and Yan, 2014). High calcium and low aluminum activities would shift the LREE and HREE reactions to the left, meaning low LREE and HREE in the clinopyroxene. The unusual nonsinusoidal shape of REE pattern (Reguir et al., 2012) and slightly higher MREE and HREE contents in clinopyroxene in Si-poor carbonatite could be then explained by the convex upward pattern of partition coefficients for REE between clinopyroxene and calciocarbonatitic melt, indicating that clinopyroxene prefers MREE relative to LREE and HREE (Prowatke and Klemme, 2006; Reguir et al., 2012; Anenburg et al., 2020). HREE are much smaller than MREE and LREE cations and can fit into the tetrahedral silicon site, $\mathrm{Ca}^{2+}+\mathrm{Si}^{4+}=2 \mathrm{HREE}^{3+}$, without requiring charge balancing with $\mathrm{Al}^{3+}$ (Anenburg et al., 2020). In apatite, the REE content correlates positively with $\mathrm{SiO}_{2}$ content (Figure 9) indicating that the REE incorporation in the Ca site was accompanied by $\mathrm{Si}$ substitution for $\mathrm{P}, \mathrm{REE}^{3+}$ $+\mathrm{Si}^{4+}=\mathrm{Ca}^{2+}+\mathrm{P}^{5+}$ (Pan and Fleet, 2002; Hammouda et al., 2010). The exchange reaction can explain the low REE content in Si-poor apatite compared to Si-rich apatite. The low LREE concentration in apatite (Figure 10) can be explained by lower partition coefficient for LREE between apatite and carbonatitic melt than MREE and HREE (convex upward pattern of partition coefficients for REE between apatite and calciocarbonatitic melt; Hammouda et al., 2010; Klemme and Dalpé, 2003) and equilibrium melt composition (Figure 12; Section 7.5; Supplementary Table S9).

Moreover, the low, subchondritic $\mathrm{Nb} / \mathrm{Ta}$ values observed in several minerals from this rock type (down to 1.9-Figures 8, 10) are opposite to the values that would be expected for differentiation from a primary melt source, either by magma unmixing (high $\mathrm{Nb} / \mathrm{Ta}$ in the carbonatitic melt, Woolley and Kjarsgaard, 2008; Mitchell, 2009) or by crystal fractionation as the latter would likely involve high $\mathrm{Nb} / \mathrm{Ta}$ melt after crystallization of $\mathrm{Nb}$-Ta-bearing minerals (e.g., $\mathrm{D}_{\mathrm{Nb} / \mathrm{Ta}}$ titanite/silicate liquid = 0.07-0.28-Prowatke and Klemme, 2006). The trace element signature of the Ihouhaouene Si-poor carbonatites rather suggests a genesis of this rock type by segregation from low$\mathrm{Nb} / \mathrm{Ta}$ melt. In this scheme, the trace element signature of the Sipoor carbonatites is the reflection of the parental melt composition, characterized by low $\mathrm{Nb} / \mathrm{Ta}$ and low LREE signature inherited from former differentiation stages involving several steps of fractional crystallization and unmixing.

\section{Further Complexity in the Carbonatite-Syenite Relationship Unveiled by Apatite and Clinopyroxene Equilibrium Melts}

As noted above, the Ihouhaouene whole-rock compositions are unlikely to represent melt compositions and therefore cannot be straightforwardly used to constrain the igneous evolution of the rock suite. To overcome this difficulty and shed light on magma compositions and their evolution, we calculated trace element compositions of equilibrium melts for apatite and clinopyroxene, using experimentally determined mineral/melt partition coefficients. For apatite, we took the apatite/carbonatite partition coefficients of Hammouda et al. (2010), who used apatite from Ihouhaouene for their experiments, and the apatite/silicate melt and apatite/trachyandesite of Prowatke and Klemme (2006) and Mahood and Stimac (1990), respectively. For clinopyroxene, we used the clinopyroxene/ carbonatite partition coefficients of Green et al. (1992) and Klemme et al. (1995) and the clinopyroxene/trachyte partition coefficients of Blundy and Dalton (2000), Mahood and Stimac (1990), and Larsen (1979) (see Supplementary Table S9 for details).

Considering the strong similarities of apatite and clinopyroxene major element compositions in carbonatites and white syenites (see Section 7.3), we made no a priori assumption on parental melt compositions in terms of major elements. Accordingly, we calculated trace element equilibrium melts using both mineral/carbonatite and mineral/silicate melt partition coefficients for each rock type. Results are illustrated in Figure 12 for chondrite-normalized REE and in Figure 13 for $\mathrm{Ce} / \mathrm{Lu}$ and $\mathrm{Nb} / \mathrm{Ta}$. Equilibrium melts show considerable variation ranges, encompassing roughly four orders of magnitude for LREE (Figure 12) and three for Ce/Lu and $\mathrm{Nb} / \mathrm{Ta}$ (Figure 13). Some systematics are however observed according to rock facies.

The white syenites and the Si-rich carbonatites are both distinguished by much higher REE content and $\mathrm{Ce} / \mathrm{Lu}$ ratios in apatite equilibrium melts than in their clinopyroxene counterparts (Figures 12, 13A,B) and high, superchondritic $\mathrm{Nb} / \mathrm{Ta}$ ratios $(>50)$ in apatite equilibrium melts compared with subchondritic $(<10)$ to superchondritic ratios in the clinopyroxene equilibrium melts (Figures 13A,B).

These results are observed with both carbonatite and silicate melt partition coefficient sets. They indicate that apatite and clinopyroxene are in disequilibrium with each other and-unexpectedly and most importantly-were most likely crystallized from different melt compositions before being eventually assembled in the studied rocks. Combined together, the $\mathrm{REE}$ and $\mathrm{Nb}-\mathrm{Ta}$ signatures suggest that apatite was equilibrated with REE-enriched carbonate melts, whereas coexisting clinopyroxene was more likely precipitated from silicate liquids. The nonprimary (evolved) composition of the latter is indicated by their low REE content compared with primary alkaline mantle melts (Sun and McDonough, 1989; Baudouin et al., 2016; Baudouin and Parat, 2020). Based on the few analyses available, this is particularly true for the white syenites, whose clinopyroxene equilibrium melts are strongly depleted in REE (Figure 12).

The Si-poor carbonatites resemble the Si-rich carbonatites (and the white syenites) in showing elevated REE contents in apatite equilibrium melts compared with clinopyroxene equilibrium melts and primary mantle melts (Figure 12), suggesting again that apatite and clinopyroxene were formed from distinct, carbonate $v s$. silicate, parental melts. At first sight, the $\mathrm{Nb} / \mathrm{Ta}$ systematics are at variance with this interpretation 
since the apatite equilibrium melt in this rock type shows a wide range of $\mathrm{Nb} / \mathrm{Ta}$ ratios $(<10-$ Figure 13A) and clinopyroxene equilibrium melts show chondritic-to-superchondritic values. In fact, this paradoxical $\mathrm{Nb}-\mathrm{Ta}$ signature may simply reflect the segregation of the carbonatite from highly, although variably, evolved silicate melts characterized by low $\mathrm{Nb} / \mathrm{Ta}$ values.

The red syenites are distinguished from the other rock types by showing roughly similar REE contents in apatite and clinopyroxene equilibrium melts, at least at first glance (Figure 12), suggesting crystallization from a common silicate melt. Moreover, these compositions are roughly comparable to those of alkaline primary melts (Sun and McDonough, 1989; Baudouin et al., 2016), indicating that the red syenites have crystallized from silicate melt that did not reach carbonate saturation and immiscibility, compared with the other facies. In detail, however, equilibrium melts calculated from apatite and clinopyroxene slightly differ from each other by higher $\mathrm{Ce} / \mathrm{Lu}$ ratios in the majority of the clinopyroxene equilibrium melts-higher in fact than in most clinopyroxene equilibrium melts from the other facies, and more comparable to the values observed in apatite equilibrium melts (Figure 13B) — somewhat higher $\mathrm{Nb} / \mathrm{Ta}$ values in the apatite equilibrium melts, compared with their clinopyroxene counterparts (Figure 13B).

These differences are not consistent with the carbonate $v s$. silicate melt duality of parental magmas as suggested for the other facies. The possibility that these subtle differences are simply a reflection of uncertainties in values of the experimental partition coefficients and/or in their application to our samples cannot be definitely excluded. However, similar differences are observed with both carbonatite and silicate melt partition coefficient sets. Hence, somewhat distinct parental melts were possibly involved in the formation of red syenite minerals as well. The comparatively less contrasted parental melt compositions may reflect the formation of this facies in an earlier differentiation stage of the complex.

Finally, trace element systematics of apatite and clinopyroxene equilibrium melts reveal that carbonatites and syenites from the Ihouhaouene complex are even more intimately related than suggested by geologic, isotopic, and petrographic evidence. All studied samples, whether carbonatites or syenites, contain mineral assemblages formed from distinct melts and as such must be viewed as 'hybrid' igneous rocks. Moreover, the compositional contrast between coexisting parental melts tends to increase with the overall degree of differentiation. While only mildly heterogeneous silicate melts were-possibly-involved in the formation of the poorly evolved red syenite, the white syenite and the carbonatites bear evidence for parental magmas of highly contrasted compositions, including coexisting carbonate and silicate melts.

The mechanism where distinct magma batches that were first differentiated from each other by fractional crystallization and liquid unmixing were thereafter mixed back after incomplete crystallization is puzzling and will clearly deserve further investigation. The key might be sought in the peculiar geodynamic setting in which the Ihouhaouene complex was emplaced, i.e., lower crust affected by a high-T thermal event (Ouzegane et al., 2003). In this context, alkaline magmas injected from the mantle into shear zones may have remained partially molten for a long period of time, allowing intermingling and mixing of magma batches and crystal mushes characterized by variable degrees of differentiation and solidification.

\section{CONCLUSION}

At first sight, the extreme diversity of trace element compositions recorded by the Ihouhaouene equilibrium melts might suggest that the complex was formed from a range of primary mantle melts generated by variable degrees of melting of a carbonated mantle source. This scheme is however at odds with the occurrence of LREE-depleted minerals in the Si-poor carbonatites, whereas the melting model would predict the opposite, i.e., selective LREE enrichment in carbonate-rich melts produced by the lowest melting degrees. In addition, variable melting degrees would hardly account for the considerable range of $\mathrm{Nb} / \mathrm{Ta}$ variations in equilibrium melt, a feature, which cannot be explained by fractional crystallization alone either.

Unmixing of carbonated melt from a silico-carbonated parental magma may account for substantial $\mathrm{Nb}-\mathrm{Ta}$ fractionation (e.g., Martin et al., 2013). However, carbonatites generated by liquid-liquid separation are expected to show elevated $\mathrm{Nb} / \mathrm{Ta}$ values. This is the case for the equilibrium melts calculated from apatite in the Si-rich carbonatites, but not for the apatite equilibrium melts in the Si-poor carbonatites. The latter are mostly characterized by low, subchondritic $\mathrm{Nb} / \mathrm{Ta}$ ratios, a signature that may be explained by segregation of carbonated melts from an evolved parental melt residual after earlier stages of unmixing.

Previous studies of volcanic alkaline/carbonatite systems have pointed out the role of fractional crystallization to reach carbonate saturation and immiscibility (Holm et al., 2006; Weidendorfer et al., 2016). Moreover, multistage processes involving alternating steps of crystal fractionation and carbonate-melt unmixing were advocated to account for the geochemical complexity of certain suites (Dawson, 1998; Baudouin et al., 2016). In the Ihouhaouene complex, a multistage process would account for

(1) the increasing importance of carbonate rock types and carbonate equilibrium melts in the presumed order of magma differentiation, i.e., from the red syenites to the Sipoor carbonatites through the white syenites and the Si-rich carbonatites;

(2) the more evolved character and wider range of equilibrium melt compositions in the white syenites and the carbonatites, compared with the red syenites;

(3) the paradoxically $\mathrm{Nb} / \mathrm{Ta}$ and low LREE signature of the Sipoor carbonatites, implying unmixing of carbonatitic melt from a differentiated parental melt.

However, additional complexity in the Ihouhaouene complex arises from the fact that all rock facies contain minerals crystallized from distinct melt types. Mineral assemblages from the most differentiated facies, in particular, include minerals 
equilibrated with both carbonate and silicate liquids. This peculiar feature is tentatively ascribed to the high thermal gradient inferred for the lower crust during emplacement of the Ihouhaouene complex, with temperatures reaching $1,000^{\circ} \mathrm{C}$ in the host granulites (Ouzegane et al., 2003). Magmas injected from the mantle into narrow shear zones may have remained partially molten for a long period of time, allowing intermingling and mixing of crystal mushes characterized by variable degrees of differentiation and solidification.

Altogether, our observations suggest an evolutionary scheme for the Ihouhaouene alkaline complex whereby slow cooling of a silicocarbonated mantle melt resulted in the segregation of both cumulus minerals (clinopyroxene, wollastonite, apatite, etc.) and immiscible silicate and carbonate melt fractions, resulting in the differentiation of the complex. This process was however counterbalanced by intermingling of partially crystallized melt fractions. This resulted in the formation of hybrid alkaline cumulates composed of disequilibrium cumulus phases and variable proportions of carbonates or K-felspar depending on the composition of the last melt fraction that was locally present upon solidification.

\section{DATA AVAILABILITY STATEMENT}

The original contributions presented in the study are included in the article/Supplementary Material, and further inquiries can be directed to the corresponding author/s.

\section{AUTHOR CONTRIBUTIONS}

AD collected the samples and carried out the microprobe, ICPMS, and LA-ICPMS analyses and defined the model for data interpretation. $\mathrm{AD}, \mathrm{FP}, \mathrm{J}-\mathrm{LB}, \mathrm{KO}$, and $\mathrm{J}-\mathrm{MD}$ contributed

\section{REFERENCES}

Adjerid, Z., Godard, G., Ouzegane, K., and Kienast, J. R. (2013). Multistage progressive evolution of rare osumilite-bearing assemblages preserved in ultrahigh-temperature granulites from In Ouzzal (Hoggar, Algeria). J. Metamorph. Geol. 31, 505-524. doi:10.1111/jmg.12031

Anenburg, M., Burnham, A. D., and Mavrogenes, J. A. (2018). REE redistribution textures in altered fluorapatite: symplectites, veins, and phosphate-silicatecarbonate assemblages from the nolans bore P-REE-Th deposit, northern territory, Australia. Can. Mineral. 56 (3), 331-354. doi:10.3749/canmin.1700038

Anenburg, M., Mavrogenes, J. A., and Bennett, V. C. (2020). The fluorapatite P-REE-Th vein deposit at nolans bore: genesis by carbonatite metasomatism. J. Petrol. 61 (1), egaa003. doi:10.1093/petrology/egaa003

Anenburg, M., and Mavrogenes, J. A. (2018). Carbonatitic versus hydrothermal origin for fluorapatite REE-Th deposits: experimental study of REE transport and crustal 'antiskarn' metasomatism. Am. J. Sci. 318 (3), 335-366. doi:10.2475/03.2018.03

Baker, M. B., and Wyllie, P. J. (1990). Liquid immiscibility in a nephelinitecarbonate system at $25 \mathrm{kbar}$ and implications for carbonatite origin. Nature 346 , $168-170$.

Barker, D. S. (2001). Calculated silica activities in carbonatite liquids. Contrib. Mineral. Petrol. 141, 704-709. doi:10.1007/s004100100281

Baudouin, C., Parat, F., Denis, C. M. M., and Mangasini, F. (2016). Nephelinite lavas at early stage of rift initiation (Hanang volcano, North Tanzanian Divergence). Contrib. Mineral. Petrol. 171, 1-20. doi:10.1007/s00410-016-1273-5 to the interpretations and writing. All authors contributed to the article and approved the submitted version.

\section{FUNDING}

This research was financially supported by the FP7-PEOPLEIRSES project MEDYNA (2014-2017) "Maghreb-EU research staff exchange on geodynamics, geohazards, and applied geology in North-west Africa," the French Embassy of Algiers through a PROFAS $\mathrm{B}+$ research grant to $\mathrm{AD}$ (2016-2017), and the French National Institute of Earth Sciences (INSU-SYSTER 2016).

\section{ACKNOWLEDGMENTS}

We thank the authorities, field guides, and drivers of Ahaggar National Park (Tamanghasset) and M. Amara for their valuable assistance during field campaigns. This work is a part ofAD's thesis (Djeddi 2019). The help of C. Martin, L. Causse, and B. Boyer for analytical work is gratefully acknowledged.We thank C. Nevado and D. Delmas for their appreciated technical assistance and Justine Villard and Rabia Benchabane for their support. We would like to thank M. Anenburg and Theo Ntaflos for very constructive reviews and $M$. Gregoire for valuable editorial handling.

\section{SUPPLEMENTARY MATERIAL}

The Supplementary Material for this article can be found online at: https:/www.frontiersin.org/articles/10.3389/feart.2020.605116/ full\#supplementary-material.

Baudouin, C., and Parat, F. (2020). Phlogopite-olivine nephelinites erupted during early stage rifting, North Tanzanian Divergence. Front. Earth Sci. 8, 277-299. doi:10.3389/feart.2020.00277

Bell, K., Kjarsgaard, B. A., and Simonetti, A. (1998). Carbonatites-into the twentyfirst century. J. Petrol. 39, 1839-1845. doi:10.1093/petroj/39.11-12.1839

Bernard-Griffiths, J., Peucat, J. J., Fourcade, S., Kienast, J. R., and Ouzegane, K. (1988). Origin and evolution of 2 Ga old carbonatite complex (Ihouhaouene, Ahaggar, Algeria): Nd and Sr isotopic evidence. Contrib. Mineral. Petrol. 100, 339-348. doi:10.1007/BF00379743

Black, R., Latouche, L., Liégeois, J. P., Caby, R., and Bertrand, J. M. (1994). PanAfrican displaced terranes in the Tuareg shield (central Sahara). J. Geol. 22, 641-644. doi:10.1130/0091-7613(1994)022<0641:PADTIT>2.3.CO;2

Blundy, J., and Dalton, J. (2000). Experimental comparison of trace element partitioning between clinopyroxene and melt in carbonate and silicate systems, and implications for mantle metasomatism. Contrib. Mineral. Petrol. 139, 356-371. doi:10.1007/s004100000139

Brooker, R. A., and Kjarsgaard, B. A. (2011). Silicate-carbonate liquid immiscibility and phase relations in the system $\mathrm{SiO}_{2}-\mathrm{Na}_{2} \mathrm{O}-\mathrm{Al}_{2} \mathrm{O}_{3}-\mathrm{CaO}-\mathrm{CO}_{2}$ at $0.1-2.5 \mathrm{GPa}$ with applications to carbonatite genesis. J. Petrol. 52, 1281-1305. doi:10.1093/ petrology/egq081

Carignan, J., Hild, P., Mevelle, G., Morel, J., Yeghicheyan, D., Br, D. R-N., et al. (2001). Routine analyses of trace elements in geological samples using flow injection and low pressure on-line liquid chromatography coupled to ICP-MS: a study of geochemical reference materials BR, DR-N, UB-N, AN-G and GH. Geostandards and Geoanalytical Research 25, 187-198. doi:10.1111/j.1751-908X.2001.tb00595.x 
Castaing, R. (1963). "X-ray microprobe techniques," in X-ray optics and X-ray microanalysis. Cambridge, MA: Academic Press, 263-277.

Chakhmouradian, A. R., Mumin, A. H., Demény, A., and Elliott, B. (2008). Postorogenic carbonatites at Eden Lake, trans-hudson orogen (northern manitoba, Canada): geological setting, mineralogy and geochemistry. Lithos. 103, 503-526. doi:10.1016/j.lithos.2007.11.004

Chen, W., and Simonetti, A. (2013). In-Situ determination of major and trace elements in calcite and apatite, and U-Pb ages of apatite from the Oka carbonatite complex: insights into a complex crystallization history, Chem. Geol. 353, 151-172. doi:10.1016/j.chemgeo.2012.04.022

Dalton, J. A., and Presnall, D. C. (1998). Carbonatitic melts along the solidus of model lherzolite in the system $\mathrm{CaO}-\mathrm{MgO}-\mathrm{Al}_{2} \mathrm{O}_{3}-\mathrm{SiO}_{2}-\mathrm{CO}_{2}$ from 3 to $7 \mathrm{GPa}$. Contrib. Mineral. Petrol. 131, 123-135.

Dalton, J. A., and Wood, B. J. (1993). The compositions of primary carbonate melts and their evolution through wallrock reaction in the mantle. Earth Planet Sci. Lett. 119, 511-525. doi:10.1016/0012-821X(93)90059-I

Dasgupta, R., and Hirschmann, M. M. (2006). Melting in the Earth's deep upper mantle caused by carbon dioxide. Nature 440, 659. doi:10.1038/ nature 04612

Dasgupta, R., Hirschmann, M. M., and Stalker, K. (2006). Immiscible transition from carbonate-rich to silicate-rich melts in the $3 \mathrm{GPa}$ melting interval of eclogite $+\mathrm{CO}_{2}$ and genesis of silica-undersaturated ocean island lavas. J. Petrol. 47, 647-671. doi:10.1093/petrology/egi088

Dawson, J. B. (1998). Peralkaline nephelinite-natrocarbonatite relationships at oldoinyo lengai, Tanzania. J. Petrol. 39, 2077-2094. doi:10.1093/petroj/39.1112.2077

Deer, W. A., Howie, R. A., and Zussman, J. (1982). Rock-forming minerals: orthosilicates. vol. 1A. New York, NY: Longman. doi:10.1002/gj.3350180308

Droop, G. T. R. (1987). A general equation for estimating Fe3+ concentrations in ferromagnesian silicates and oxides from microprobe analyses, using stoichiometric criteria. Mineral. Mag. 51, 431-435. doi:10.1180/minmag. 1987.051.361.10

Drüppel, K., Hoefs, J., and Okrusch, M. (2004). Fenitizing processes induced by ferrocarbonatite magmatism at swartbooisdrif, NW Namibia. J. Petrol. 46, 377-406. doi:10.1093/petrology/egh081

Dygert, N., and Yan, L. (2014). Temperatures and cooling rates recorded in REE in coexisting pyroxenes in ophiolitic and abyssal peridotites. Earth Planet. Sci. Lett. 420, 151-161. doi:10.1016/j.epsl.2015.02.042

Eby, G. N. (1975). Abundance and distribution of the rare-earth elements and yttrium in the rocks and minerals of the Oka carbonatite complex, Quebec. Geochimica et Cosmochimica Acta. 39, 597-620.

Elliott, H. A. L., Wall, F., Chakhmouradian, A. R., Siegfried, P. R., Dahlgren, S., Weatherley, S., et al. (2018). Fenites associated with carbonatite complexes: a review. Ore Geol. Rev. 93, 38-59. doi:10.1016/j.oregeorev. 2017.12.003

Fourcade, S., Kienast, J. R., and Ouzegane, K. (1996). Metasomatic effects related to channelled fluid streaming through deep crust: fenites and associated carbonatites (In Ouzzal Proterozoic granulites, Hoggar, Algeria). J. Metamorph. Geol. 14, 763-781. doi:10.1111/j.1525-1314.1996.00055.x

Freestone, I. C., and Hamilton, D. L. (1980). The role of liquid immiscibility in the genesis of carbonatites-an experimental study. Contrib. Mineral. Petrol. 73, 105-117. doi:10.1007/BF00371385

Giebel, R. J., Parsapoor, A., Walter, B. F., Braunger, S., Marks, M. A. W., Wenzel, T., et al. (2019). Evidence for magma-wall rock interaction in carbonatites from the Kaiserstuhl Volcanic Complex (Southwest Germany). J. Petrol. 60, 1163-1194. doi:10.1093/petrology/egz028/5488968

Gittins, J., and Harmer, R. E. (1997). What is ferrocarbonatite? A revised classification. J. Afr. Earth Sci. 25, 159-168. doi:10.1016/S0899-5362(97) 00068-7

Goldoff, B., Webster, J. D., and Harlov, D. E. (2012). Characterization of fluorchlorapatites by electron probe microanalysis with a focus on time-dependent intensity variation of halogens. Am. Mineral. 97 (7), 1103-1115. doi:10.2138/ am.2012.3812

Green, T. H., Adam, J., and Sie, S. H. (1992). Trace element partitioning between silicate minerals and carbonatite at $25 \mathrm{kbar}$ and application to mantle metasomatism. Mineral. Petrol. 46, 179-184.

Griffin, W. L., Powell, W. J., Pearson, N. J., and O’Reilly, S. Y. (2008). “GLITTER: data reduction software for laser ablation ICP-MS. Laser ablation-ICP-MS in the earth sciences," in Mineralogical association of Canada short course series (Quebec), Vol. 40, 204-207.

Gudfinnsson, G. H., and Presnall, D. C. (2005). Continuous gradations among primary carbonatitic, kimberlitic, melilititic, basaltic, picritic, and komatiitic melts in equilibrium with garnet lherzolite at 3-8 GPa. J. Petrol. 46, 1645-1659. doi:10.1093/petrology/egi029

Guiraud, M., Kienast, J. R., and Ouzegane, K. (1996). Corundum-quartz bearing assemblage in the Ihouhaouene area in Ouzzal, Algeria. J. Metamorph. Geol. 14, 755-761. doi:10.1111/j.1525-1314.1996.00046.x

Hamilton, D. L., Bedson, P., and Esson, J. (1989). “The behaviour of trace elements in the evolution of carbonatites," in Carbonatites, genesis and evolution. Editor K. Bell (London: Unwin and Hyman), 405-427.

Hammouda, T. (2003). High-pressure melting of carbonated eclogite and experimental constraints on carbon recycling and storage in the mantle. Earth Planet. Sci. Lett. 214, 357-68. doi:10.1016/S0012-821X(03)00361-3

Hammouda, T., Chantel, J., and Devidal, J. L. (2010). Apatite solubility in carbonatitic liquids and trace element partitioning between apatite and carbonatite at high pressure. Geochem. Cosmochim. Acta. 74, 7220-7235. doi:10.1016/j.gca.2010.09.032

Hammouda, T., and Shantanu, K. (2015). Melting in the mantle in the presence of carbon: review of experiments and discussion on the origin of carbonatites. Chem. Geol. Elsevier B.V. 418, 171-88. doi:10.1016/j. chemgeo.2015.05.018

Holm, P. M., Wilson, J. R., Christensen, B. P., Hansen, L., Hansen, S. L., Hein, K. M., et al. (2006). Sampling the Cape Verde mantle plume: evolution of melt composition on Santo Antão, Cape Verde Islands. J. Petrol. 47, 145-189. doi:10. 1093/petrology/egi071

Jones, A. P., and Wyllie, P. J. (1985). Paragenetic trends of oxide minerals in carbonate-rich kimberlites with new analyses from the Benfontein sill, South Africa. J. Petrol. 26, 210-222.

Jones, J. H., Walker, D., Pickett, D. A., Murrell, M. T., and Beattie, P. (1995). Experimental investigations of the partitioning of $\mathrm{Nb}, \mathrm{Mo}, \mathrm{Ba}, \mathrm{Ce}, \mathrm{Pb}, \mathrm{Ra}, \mathrm{Th}$, $\mathrm{Pa}$, and $\mathrm{U}$ between immiscible carbonate and silicate liquids. Geochem. Cosmochim. Acta. 59, 1307-1320. doi:10.1016/0016-7037(95)00045-2

Jung, S., Pfänder, J. A., Brügmann, G., and Stracke, A. (2005). Sources of primitive alkaline volcanic rocks from the central European volcanic province (rhön, Germany) inferred from Hf, Os and Pb isotopes. Contrib. Mineral. Petrol. 150, 546-559. doi:10.1007/s00410-005-0029-4

Kjarsgaard, B. A., Hamilton, D. L., and Peterson, T. D. (1995). "Peralkaline nephelinite/carbonatite liquid immiscibility: comparison of phase compositions in experiments and natural lavas from Oldoinyo Lengai," in Carbonatites volcanism-Oldoinyo Lengai and petrogenesis of natrocarbonatites. IAVCEI proceedings in volcanology. Editors K. Bell and J. Keller, Vol. 4, 163-190. doi:10.1007/978-3-642-79182-6_13

Kjarsgaard, B. A., and Peterson, T. (1991). Nephelinite-carbonatite liquid immiscibility at Shombole volcano, East Africa: petrographic and experimental evidence. Mineral. Petrol. 43, 293-314.

Klemme, S., and Dalpé, C. (2003). Trace-element partitioning between apatite and carbonatitic melt. Am. Mineral. 88, 639-646. doi:10.2138/am-2003-0417

Klemme, S., van der Laan, S. R., Foley, S. F., and Günther, D. (1995). Experimentally determined trace and minor element partitioning between climopyroxene and carbonatite melt under upper mantle conditions. Earth Planet Sci. Lett. 133, 439-448. doi:10.1016/0012-821X(95)00098-W

Koster van Groos, A. F., and Wyllie, P. J. (1968). Liquid immiscibility in the join $\mathrm{NaAlSi}_{3} \mathrm{O}_{8}-\mathrm{Na}_{2} \mathrm{CO}_{3}-\mathrm{H}_{2}$ O. Am. J. Sci. 266, 932-967. doi:10.2475/ajs.273.6.465

Kresten, P. (1988). The chemistry of fenitization: examples from Fen, Norway. Chem. Geol. 68, 329-349. doi:10.1016/0009-2541(88)90030-7

Lapin, A. V., and Ploshko, V. V. (1988). Rock-association and morphological types of carbonatite and their geotectonic environments. Int. Geol. Rev. 30, 390-396. doi:10.1080/00206818809466019

Larsen, L. M. (1979). Distribution of REE and other trace elements between phynocrysts and peralkaline undersaturated magmas, explified by rocks from the Gardar igneous province, South Greenland. Lithos 12, 303-315. doi:10. 1016/0024-4937(79)90022-7

Le Maitre, R. W., Bateman, P., Dubek, A., Keller, J., Lameyre, J., Le Bas, M. J., et al. (1989). A classification of igneous rocks and glossary of terms: recommendations of the international union of geological Sciences subcommission on the systematics of igneous rocks. Oxford, NY: Blackwell. 
Lorenz, M., Altenberger, U., Trumbull, R. B., Lira, R., López de Luchi, M., Günter, C., et al. (2019). Chemical and textural relations of britholite- and apatite-group minerals from hydrothermal REE mineralization at the Rodeo de los Molles deposit, Central Argentina. Am. Mineral. 104, 1840-1850. doi:10.2138/am2019-6969

Mahood, G. A., and Stimac, J. A. (1990). Trace-element partitioning in pantellerites and trachytes. Geochem. Cosmochim. Acta. 54, 2257-2276. doi:10.1016/00167037(90)90050-U

Malvoisin, B., Chopin, C., Brunet, F., and Galvez, M. E. (2012). Low-temperature wollastonite formed by carbonate reduction: a marker of serpentinite redox conditions. J. Petrol. 53, 159-176. doi:10.1093/petrology/egr060

Martin, L. H. J., Schmidt, M. W., Mattsson, H. B., and Guenther, D. (2013). Element partitioning between immiscible carbonatite and silicate melts for dry and H2O-bearing systems at 1-3 GPa. J. Petrol. 54, 2301-2338. doi:10.1093/ petrology/egt 048

Martins, T., Couëslan, C. G., and Böhm, C. O. (2011). The Burntwood Lake alkali-feldspar syenite revisited, west-central Manitoba (part of NTS 63N8). In Report of activities 2011, manitoba innovation, energy and mines, manitoba geological survey, 79-85.

Mitchell, R. H. (2009). Peralkaline nephelinite-natrocarbonatite immiscibility and carbonatite assimilation at Oldoinyo Lengai, Tanzania. Contrib. Mineral. Petrol. 158, 589-598. doi:10.1007/s00410-009-0398-1

Morimoto, N. (1988). Nomenclature of pyroxenes. Mineral. Petrol. 39 (1), $55-76$.

Novella, D., Keshav, S., Gudfinnsson, G. H., and Ghosh, S. (2014). Melting phase relations of model carbonated peridotite from 2 to $3 \mathrm{GPa}$ in the system $\mathrm{CaO}$ $\mathrm{MgO}-\mathrm{Al}_{2} \mathrm{O}_{3}-\mathrm{SiO}_{2}-\mathrm{CO}_{2}$ and further indication of possible unmixing between carbonatite and silicate liquids. J. Geophys. Res. Solid Earth 119, 2780-2800. doi:10.1002/2013JB010913

Oberti, R., Ottolini, L., Della Ventura, G., and Parodi, G. C. (2001). On the symmetry and crystal chemistry of britholite: new structural and microanalytical data. Am. Mineral. 86, 1066-1075. doi:10.2138/am-2001-8-913

Olin, P. H., and Wolff, J. A. (2010). Rare earth and high field strength element partitioning between iron-rich clinopyroxenes and felsic liquids. Contrib. Mineral. Petrol. 160 (5), 761-775.

Olson, J. C., Shawe, D. R., Pray, L. C., and Sharp, W. N. (1954). Rare-earth mineral deposits of the Mountain Pass district, San Bernardino County, California. US Geol Surv Prof pap 261, 75.

Ouzegane, K., Fourcade, S., Kienast, J., and Javoy, M. (1988). New carbonatite complexes in the archaean In'Ouzzal nucleus (ahaggar, Algeria): mineralogical and geochemical data. Contrib. Mineral. Petrol. 98, 277-292.

Ouzegane, K., Kienast, J. R., Bendaoud, A., and Drareni, A. (2003). A review of archaean and paleoproterozoic evolution of the In Ouzzal granulitic terrane (western hoggar, Algeria). J. Afr. Earth Sci. 37, 207-227.

Pan, Y., and Fleet, M. E. (2002). "Composition of the apatite-group minerals: substitution mechanisms and controlling factors," Rev. Mineral Geochem 48, 13-49. doi:10.2138/rmg.2002.48.2

Pearce, N. J. G., Perkins, W. T., Westgate, J. A., Gorton, M. P., Jackson, S. E., and NealChenery, G. R. S. P. (1997). A compilation of new and published major and trace element data for NIST SRM 610 and NIST SRM 612 glass reference materials. Geostand. Newsl. 21 (1), 115-144. doi:10.1111/j.1751-908X.1997.tb00538.x

Peucat, J. J., Capdevila, R., Drareni, A., Choukroune, P., Fanning, M., BernardGriffiths, J., et al. (1996). Major and trace element geochemistry and isotope Sr, $\mathrm{Nd}, \mathrm{Pb}, \mathrm{O}$ systematics of an Archaean basement involved in a $2.0 \mathrm{Ga}$ VHT $1000^{\circ} \mathrm{C}$ metamorphic event: in Ouzzal massif, Hoggar, Algeria. J. Metamorph. Geol. 14, 667-692. doi:10.1111/j.1525-1314.1996.00054.x

Prowatke, S., and Klemme, S. (2006). Trace element partitioning between apatite and silicate melts. Geochem. Cosmochim. Acta. 70, 4513-4527. doi:10.1016/j. gca.2006.06.162

Ramasamy, R. (1986). Titanium-bearing garnets from alkaline rocks of carbonatite complex of Tiruppattur, Tamil Nadu. Curr Sci. 55, 1026-1029. doi:10.1016/j.gca.2006.06.162
Reguir, E. P., Chakhmouradian, A. R., Pisiak, L., Halden, N. M., Yang, P., Xu, C., et al. (2012). Trace-element composition and zoning in clinopyroxene and amphibole-group minerals: implications for element partitioning and evolution of carbonatites. Lithos 128 (131), 27-45. doi:10.1016/j.lithos.2011.10.003

Saha, A., Ray, J., Ganguly, S., and Chatterjee, N. (2011). Occurrence of melanite garnet in syenite and ijolite - melteigite rocks of Samchampi - samteran alkaline complex, Mikir Hills, Northeastern India. Curr. Sci. 101, 1-10.

Smith, M. P., Henderson, P., and Campbell, L. S. (2000). Fractionation of the REE during hydrothermal processes: constraints from the Bayan Obo Fe-REE-Nb deposit, Inner Mongolia, China. Geochimica et Cosmochimica Acta. 64 (18), 3141-3160

Stoppa, F., Rosatelli, G., Wall, F., and Jeffries, T. (2005). Geochemistry of carbonatitesilicate pairs in nature: a case history from Central Italy. Lithos. 85, 26-47.

Streckeisen, A. (1974). Classification and nomenclature of plutonic rocks recommendations of the IUGS subcommission on the systematics of Igneous Rocks. Geol. Rundsch. 63, 773-786.

Sun, S., and McDonough, W. F. (1989). Chemical and isotopic systematics of oceanic basalts: implications for mantle composition and processes. Geol. Soc. London Spec. Publ. 42, 313-345.

Taylor, S. R., and McLennan, S. M. (1985). The continental crust: its composition and evolution. Oxford, NY: Blackwells Scientific, 312.

Verhulst, A., Balaganskaya, E., Kirnarsky, Y., and Demaiffe, D. (2000). Petrological and geochemical (trace elements and $\mathrm{Sr}-\mathrm{Nd}$ isotopes) characteristics of the Paleozoic Kovdor ultramafic, alkaline and carbonatite intrusion (Kola Peninsula, NW Russia). Lithos. 51, 1-25.

Walter, B. F., Parsapoor, A., Braunger, S., Marks, M. A. W., Wenzel, T., Martin, M., et al. (2018). Pyrochlore as a monitor for magmatic and hydrothermal processes in carbonatites from the Kaiserstuhl volcanic complex, SW Germany. Chem. Geol. 498, 1-16. doi:10.1016/j.chemgeo.2018.08.008

Weidendorfer, D., Schmidt, M. W., and Mattsson, H. B. (2016). Fractional crystallization of Si-undersaturated alkaline magmas leading to unmixing of carbonatites on Brava Island (Cape Verde) and a general model of carbonatite genesis in alkaline magma suites. Contrib. Mineral. Petrol. 171, 1-29. doi:10. 1007/s00410-016-1249-5

Woolley, A. R. (2003). Igneous silicate rocks associated with carbonatites: their diversity, relative abundances and implications for carbonatite genesis. Periodico di Mineralogia 72, 9-17.

Woolley, A. R., and Kempe, D. R. C. (1989). "Carbonatites: nomenclature, average chemical compositions, and element distribution," in Carbonatites, genesis and evolution. Editor K. Bell (Unwin and Hyman), 1-14.

Woolley, A. R., and Kjarsgaard, B. A. (2008). Paragenetic types of carbonatite as indicated by the diversity and relative abundances of associated silicate rocks: evidence from a global database. Can. Mineral. 46 (4), 741-752. doi:10.3749/ canmin.46.4.741

Wyllie, P. J., and Huang, W. L. (1975). Peridotite, Kimberlite and carbonatite explained in the system $\mathrm{CaO}-\mathrm{MgO}-\mathrm{SiO}_{2}-\mathrm{CO}_{2}$. Geology 3, 621-624. doi:10.1130/ 0091-7613(1975)3<621:PKACEI >2.0.CO;2

Wyllie, P. J., and Lee, W. J. (1998). Model system controls on conditions for formation of magnesiocarbonatite and calciocarbonatite magmas from the mantle. J. Petrol. 39, 1885-1893. doi:10.1093/petroj/39.11-12.1885

Conflict of Interest: The authors declare that the research was conducted in the absence of any commercial or financial relationships that could be construed as a potential conflict of interest.

Copyright $\odot 2021$ Djeddi, Parat, Bodinier, Ouzegane and Dautria. This is an openaccess article distributed under the terms of the Creative Commons Attribution License (CC BY). The use, distribution or reproduction in other forums is permitted, provided the original author(s) and the copyright owner(s) are credited and that the original publication in this journal is cited, in accordance with accepted academic practice. No use, distribution or reproduction is permitted which does not comply with these terms. 